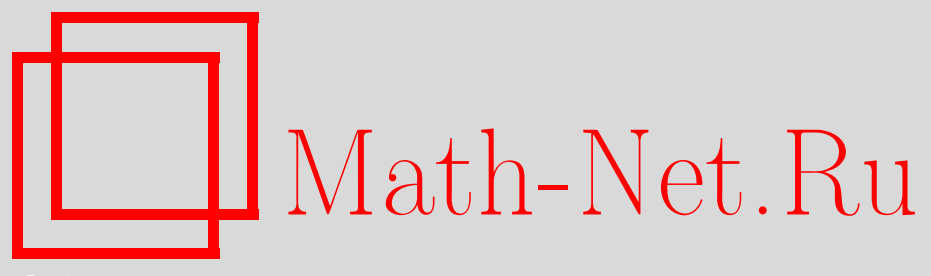

А. Н. Андрианов, Дзета-функции ортогональных групп целочисленных положительно-определенных квадратичных форм, УМH, 2006, том 61, выпуск 6, 3-44

DOI: https://doi.org/10.4213/rm4952

Использование Общероссийского математического портала Math-Net.Ru подразумевает, что вы прочитали и согласны с пользовательским соглашением http: //www. mathnet.ru/rus/agreement

Параметры загрузки:

IP: 18.209 .158 .208

26 апреля 2023 г., 12:20:22

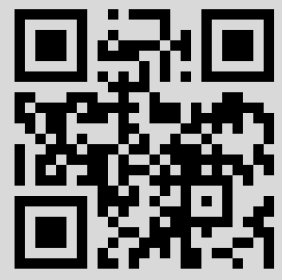


УДК $511.331+511.38$

\title{
Дзета-функции ортогональных групп целочисленных положительно-определенных квадратичных форм
}

\author{
А. Н. Андрианов
}

В обзоре рассмотрены представления колец Гекке-Шимуры произвольных целочисленных положительно-определенных квадратичных форм на пространствах полиномиальных гармонических векторов и исследован вопрос об одновременной диагонализации соответствующих операторов Гекке. Получены точные соотношения между дзета-функциями квадратичных форм от 2 до 4 переменных, отвечающими гармоническим собственным векторам родов 1 и 2, и дзета-функциями Гекке и Андрианова тетарядов, взвешенных этими векторами соответственно. Аналогичные вопросы для одноклассных квадратичных форм были рассмотрены ранее в работе [1]. Общая ситуация рассматривалась в работе [2].

Библиография: 22 названия.

\section{СОДЕРЖАНИЕ}

$\S 1$. Об ортогональных дзета-функциях ........................ 3

$\S 2$ Кольца классов автоморфов целочисленных квадратичных форм ... 6

$\S 3$. Представления на гармонических векторах.................. 19

$\S 4$. Действие операторов Гекке на гармонические тета-суммы .......... 24

$\S 5$. Бинарные формы фундаментальных дискриминантов ........... 35

Список литературы ...................................... 43

\section{§ 1. Об ортогональных дзета-функциях}

Основные объекты и инструменты исследования арифметической теории представлений групп Ли - дзета-функции дискретных подгрупп - естественно возникают при рассмотрении представлений соответствующих алгебр ГеккеШимуры на подходящих пространствах автоморфных форм (автоморфные представления). В самом известном случае дзета-функций модулярных форм рассматриваются представления алгебр Гекке-Шимуры подгрупп целочисленных симплектических групп $\mathrm{Sp}_{n}(\mathbb{Z})$ на пространствах голоморфных модулярных форм, задаваемые операторами Гекке.

Работа выполнена при поддержке Российского фонда фундаментальных исследований (грант № 05-01-00930).

(C) А. Н. Андрианов, 2006 
Настоящий обзор посвящен систематическому изучению дзета-функций ортогональных групп целочисленных положительно-определенных квадратичных форм, начатому в работе [1] для случая одноклассных форм. В ситуации ортогональных групп алгебры Гекке-Шимуры возникают как кольца классов автоморфов подходящих систем целочисленных квадратичных форм. В простейшем случае одноклассных квадратичных форм, когда, например, род формы состоит из единственного класса относительно целочисленной эквивалентности, это просто кольца классов автоморфов таких форм. (Целочисленный собственный) автоморф неособой целочисленной квадратичной формы $\mathbf{q}(X)$ от $m$ переменных это, по определению, целочисленная $(m \times m)$-матрица $D$ с положительным определителем, удовлетворяющая условию

$$
\mathbf{q}(D X)=\mu \mathbf{q}(X) \quad\left({ }^{t} X=\left(x_{1}, \ldots, x_{m}\right)\right),
$$

где $\mu=\mu(D)$ - мультипликатор автоморфа. Все автоморфы образуют полугруппу $\mathbf{A}=A(\mathbf{q})$ - полугруппу автоморфов формы $\mathbf{q}$, а автоморфы мультипликатора $\mu=1$ образуют группу $\mathbf{E}=E(\mathbf{q})$ - группу (собственных) единии, формы q. Кольцо Гекке-Шимурь, или кольцо классов автоморфов, формы $\mathbf{q}$ над $\mathbb{Z}$ состоит из всех конечных формальных линейных комбинаций с целочисленными коэффициентами символов $(\mathbf{E} D \mathbf{E})=\tau(D)$, взаимно однозначно соответствующих двусторонним классам смежности $\mathbf{E} D \mathbf{E}$ полугруппы $\mathbf{A}$ по подгруппе $\mathbf{E}$, называемым ниже двойнъми классами,

$$
\mathcal{H}=\mathcal{H}(\mathbf{q})=\left\{\tau=\sum_{\alpha} a_{\alpha} \tau\left(D_{\alpha}\right) \text { (формальные конечные) } \mid a_{\alpha} \in \mathbb{Z}, D_{\alpha} \in \mathbf{A}\right\},
$$

где произведение двух двойных классов определяется формулой

$$
\tau(D) \tau\left(D^{\prime}\right)=\sum_{\mathbf{E} D^{\prime \prime} \mathbf{E} \subset \mathbf{E} D \mathbf{E} D^{\prime} \mathbf{E}} c\left(D, D^{\prime} ; D^{\prime \prime}\right) \tau\left(D^{\prime \prime}\right)
$$

и $c\left(D, D^{\prime} ; D^{\prime \prime}\right)$ обозначает число пар представителей $D_{i} \in \mathbf{E} \backslash \mathbf{E} D \mathbf{E}, \quad D_{j}^{\prime} \in$ $\mathbf{E} \backslash \mathbf{E} D^{\prime} \mathbf{E}$, удовлетворяющих условию $D_{i} D_{j}^{\prime} \in \mathbf{E} D^{\prime \prime}$. Если форма $\mathbf{q}$ не является одноклассной, кольцо $\mathcal{H}$ следует заменить более сложной конструкцией матричного кольца Гекке-Шимуры, которая будет объяснена в следующем параграфе. В одноклассном случае суммы двойных классов фиксированных мультипликаторов,

$$
\tau(\mu)=\sum_{D \in \mathbf{E} \backslash \mathbf{A} / \mathbf{E}, \mu(D)=\mu} \tau(D) \in \mathcal{H},
$$

удовлетворяют простым мультипликативным соотношениям

$$
\tau(\mu) \tau(\nu)=\tau(\nu) \tau(\mu)=\tau(\mu \nu)
$$

если $\mu$ и $\nu$ взаимно просты и $\mu$ или $\nu$ взаимно просты с определителем формы q. Отсюда следует, что формальный ряд Дирихле с коэффициентами $\tau(1), \tau(2), \ldots$, где класс $\tau(1)=\tau\left(1_{m}\right)$ является единичным элементом кольца $\mathcal{H}$, может быть разложен в формальное эйлерово произведение:

$$
\sum_{\mu=1}^{\infty} \frac{\tau(\mu)}{\mu^{s}}\left(\sum_{\nu \mid(\operatorname{det} \mathbf{q})^{\infty}} \frac{\tau(\nu)}{\nu^{s}}\right) \prod_{p \nmid \operatorname{det} \mathbf{q}} \sum_{\delta=0}^{\infty} \tau\left(p^{\delta}\right) p^{-\delta s},
$$


при этом мы рассматриваем символ $\mu^{s}$ просто как формальный квазихарактер мультипликативной полугруппы $\mathbb{N}$ целых положительных чисел, а $\nu$ и $p$ пробегают все положительные целые числа, делящие некоторые степени числа $\operatorname{det} \mathbf{q}$, и простые числа, не делящие число $\operatorname{det} \mathbf{q}$, соответственно. В работе [3] в общей ситуации было выдвинуто предположение, что для каждого простого числа $p$, не делящего определитель $\operatorname{det} \mathbf{q}$, формальный степенной ряд с коэффициентами $\tau(1), \tau(p), \tau\left(p^{2}\right), \ldots$ является (формально) рациональной дробью над кольцом $\mathcal{H}=\mathcal{H}(\mathbf{q})$ со знаменателем степени $2^{k}$ и числителем степени самое большее $2^{k}-2$, где целое число $k$ определяется из условия, что число $m$ переменных формы q имеет вид $2 k-1$ или $2 k$ :

$$
\sum_{\delta=0}^{\infty} \tau\left(p^{\delta}\right) t^{\delta}=R_{p}(t)^{-1} S_{p}(t)
$$

где

$$
\begin{aligned}
R_{p}(t) & =\tau(1)+\sum_{1 \leqslant i \leqslant 2^{k}} \rho_{i} t^{i}, \\
S_{p}(t) & =\sum_{0 \leqslant j \leqslant 2^{k}-2} \sigma_{j} t^{j}
\end{aligned}
$$

с $\rho_{i}=\rho_{i}(p), \sigma_{j}=\sigma_{j}(p) \in \mathcal{H}(\mathbf{q})$. В этом случае мы будем говорить, что формальный степенной ряд над кольцом $\mathcal{H}(\mathbf{q})$ вида

$$
Z_{p}(t, \mathbf{q})=R_{p}(t)^{-1}
$$

является локальным дзета-рядом формы q. В [3] было доказано, в частности, что это предположение справедливо для квадратичных форм от $=2,3$ и 4 переменных.

Предположим теперь, что задано комплексное представление $\mathcal{H}(\mathbf{q}) \ni \tau \mapsto \mid \tau$ кольца $\mathcal{H}(\mathbf{q})$ на некотором линейном пространстве и что $P$ - некоторая общая собственная функция, так что $P \mid \tau=\lambda(\tau) P$ для всех $\tau \in \mathcal{H}(\mathbf{q})$ с собственными числами $\lambda(\tau)$. Как пространства представлений в этом случае могут фигурировать пространства гармонических многочленов, отвечающих рассматриваемой квадратичной форме. Тогда, по аналогии с теорией дзета-функций зигелевых модулярных форм, можно рассмотреть степенной ряд

$$
Z_{p}(t, P)=\left(1+\sum_{1 \leqslant i \leqslant 2^{k}} \lambda\left(\rho_{i}\right) t^{i}\right)^{-1}
$$

и эйлерово произведение

$$
Z^{*}(s, P)=\prod_{p \nmid \operatorname{det} \mathbf{q}} Z_{p}\left(p^{-s}, P\right),
$$

которые естественно назвать локальной и (регулярной) глобальной ортогональной дзета-функциями формы q соответственно, отвечающими собственной

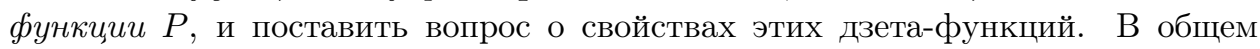
случае представление на гармонических формах заменяется представлением 
на гармонических векторах. На естественный вопрос о существовании связей между дзета-функциями, отвечающими различным типам дискретных подгрупп, мы получим ниже частичный положительный ответ. Будет показано, что в некоторых случаях ортогональные дзета-функции могут быть явно выражены через спинорные дзета-функции подходящих зигелевых модулярных форм.

В $\S 2$ содержится обзор основных определений и свойств колец классов автоморфов. В $\S 3$ рассматриваются стандартные представления колец классов автоморфов на пространствах гармонических векторов и исследуется вопрос о существовании собственных векторов. В 4 , в частности, получены явные выражения дзета-функций положительно-определенных квадратичных форм от $m=2$ и 4 переменных, отвечающих собственным векторам, через дзетафункции Гекке, если $m=2$, и дзета-функции Андрианова модулярных форм рода 2 , если $m=4$, гармонических тета-рядов с этими гармоническими собственными векторами в качестве коэффициентов (теоремы 4.2 и 4.3 соответственно). В $\S 5$ показано, что в случае бинарных квадратичных форм фундаментальных дискриминантов соответствующие глобальные дзета-функции фактически совпадают с дзета-функциями соответствующих квадратичных полей с подходящими характерами Гекке.

Вряд ли можно сомневаться, что представленные в настоящем обзоре результаты непростых вычислений являются отражением гораздо более общих связей автоморфных представлений алгебр Гекке-Шимуры ортогональной и симплектической групп над глобальными полями. Формализм преобразований, выражающих образы гармонических тета-рядов под действием операторов Гекке через действие колец классов автоморфов на их гармонические коэффициенты, и стоящие за ним прямые связи между кольцами Гекке-Шимуры симплектических групп и кольцами классов автоморфов ортогональных групп могут представить базовые средства для исследования этих связей (см., например, [4]-[6]). Тем не менее, прямые подходы к ортогональным дзета-функциям, не основанные на сведении к симплектическому случаю, также представляют значительный интерес.

Обозначения. Как обычно, мы обозначаем через $\mathbb{N}, \mathbb{Z}, \mathbb{Q}$ и $\mathbb{C}$ множество положительных целых рациональных чисел, кольцо целых рациональных чисел, поле рациональных чисел и поле комплексных чисел соответственно.

Если $\mathbb{A}$ - некоторое множество, то $\mathbb{A}_{n}^{m}$ обозначает множество всех матриц размера $m \times n$ с элементами из $\mathbb{A}$. Если $\mathbb{A}-$ кольцо с единичным элементом, то $1_{n}$ обозначает единичный элемент кольца $\mathbb{A}_{n}^{n}$. Результат транспонирования матрицы $M$ обозначается через ${ }^{t} M$. Для двух матриц $S$ и $N$ соответствующих размеров мы пишем

$$
S[N]={ }^{t} N S N
$$

\section{§ 2. Кольца классов автоморфов целочисленных квадратичных форм}

В этом параграфе мы определим кольца классов автоморфов целочисленных неособых квадратичных форм и рассмотрим основные свойства этих колец. Мы 
следуем, в основном, схеме, изложенной в работе [7], но заменяем системы представителей из классов целочисленной эквивалентности четных матриц одинакового размера, сигнатуры, делителя, ступени и определителя, рассматривавшиеся в [7], на более естественные системы представителей из классов собственной целочисленной эквивалентности, содержащихся в собственных классах подобия четных матриц.

Сначала мы определим абстрактные кольца, которые можно рассматривать как матричные обобщения колец двойных классов Гекке-Шимуры. Предположим, что заданы мультипликативная группа $G$, конечное множество ее подгрупп $\Lambda_{1}, \ldots, \Lambda_{h}$ и подмножества $\Sigma_{i j} \subset G$ для всех пар индексов $i, j=1, \ldots, h$. Мы будем говорить, что система

$$
\mathcal{S}=\left(\Lambda_{1}, \ldots, \Lambda_{h} ; \Sigma_{11}, \Sigma_{12}, \ldots, \Sigma_{h h}\right)
$$

является $h s$-системой, если выполняются следующие три условия:

(1) $\Sigma_{i j} \Sigma_{j k} \subset \Sigma_{i k}$ для $i, j, k=1, \ldots, h$;

(2) $\Lambda_{i} \subset \Sigma_{i i}$ для $i=1, \ldots, h$;

(3) каждый двойной класс $\Lambda_{i} g \Lambda_{j}$ с $g \in \Sigma_{i j}$ является объединением конечного числа левых классов (смежности) $\Lambda_{i} g^{\prime}$.

Легко проверить, что, поскольку $G$ группа, каждый двойной класс $\Lambda_{i} g \Lambda_{j}$ с $g \in \Sigma_{i j}$ является также объединением конечного числа правых классов (смежности) $g^{\prime} \Lambda_{j}$, и разложение двойного класса $\Lambda_{i} g \Lambda_{j}$ в объединение непересекающихся левых классов по модулю $\Lambda_{i}$ (соответственно правых классов по модулю $\left.\Lambda_{j}\right)$ может быть представлено соответственно в виде

$$
\Lambda_{i} g \Lambda_{j}=\left\{\begin{array}{l}
\bigcup_{\lambda \in\left(\Lambda_{j} \cap g^{-1} \Lambda_{i} g\right) \backslash \Lambda_{j}} \Lambda_{i} g \lambda, \\
\bigcup_{\lambda \in \Lambda_{i} /\left(\Lambda_{i} \cap g \Lambda_{j} g^{-1}\right)} \lambda g \Lambda_{j} .
\end{array}\right.
$$

Мы будем говорить, что некоторая $h s$-система (2.1) является ручной, если каждый из двойных классов $\Lambda_{i} g \Lambda_{j}$ с $g \in \Sigma_{i j}$ содержит одинаковое число различных правых классов $g^{\prime} \Lambda_{j}$ и левых классов $\Lambda_{i} g^{\prime}$. Так как $G$ группа, то, согласно (2.2), это условие означает, что

$$
\begin{aligned}
& {\left[\Lambda_{i}:\left(\Lambda_{i} \cap g \Lambda_{j} g^{-1}\right)\right]=\left[\Lambda_{j}:\left(\Lambda_{j} \cap g^{-1} \Lambda_{i} g\right)\right]} \\
& \quad=\left[g \Lambda_{j} g^{-1}:\left(\Lambda_{i} \cap g \Lambda_{j} g^{-1}\right)\right] \quad\left(\text { для всех } i, j=1, \ldots, h \text { и } g \in \Sigma_{i j}\right) .
\end{aligned}
$$

ЛЕмма 2.1. (1) hs-система (2.1) является ручной в том и только том случае, если каждый двойной класс $\Lambda_{i} g \Lambda_{j}$ с $g \in \Sigma_{i j}$ содержит общую систему представителей из левых классов по модулю $\Lambda_{i}$ и правых классов по модулю $\Lambda_{j}$.

(2) Если все группы $\Lambda_{1}, \ldots, \Lambda_{h}$ конечны, то hs-система (2.1) является ручной в том и только том случае, если эти группы имеют равные порядки:

$$
\#\left(\Lambda_{1}\right)=\cdots=\#\left(\Lambda_{h}\right)
$$

ДокАзАтельство. Утверждение (1) следует из очевидного наблюдения, что каждый левый класс $\Lambda_{i} g \lambda$ с $\lambda \in \Lambda_{j}$ имеет непустое пересечение с каждым 
правым классом $\mu g \Lambda_{j}$ с $\mu \in \Lambda_{i}$, поскольку элемент $\mu g \lambda$ содержится в обоих классах. Если группы $\Lambda_{i}$ и $\Lambda_{j}$ конечны, то множество $\Lambda_{i} g \Lambda_{j}$ тоже конечно и

$$
\#\left(\Lambda_{i} g \Lambda_{j}\right)=\#\left(\Lambda_{i}\right) \#\left(\Lambda_{i} \backslash \Lambda_{i} g \Lambda_{j}\right)=\#\left(\Lambda_{i} g \Lambda_{j} / \Lambda_{j}\right) \#\left(\Lambda_{j}\right),
$$

так что равенства (2.3) эквивалентны условиям $\#\left(\Lambda_{i}\right)=\#\left(\Lambda_{j}\right)$. Лемма 2.1 доказана.

Для данной $h s$-системы $(2.1)$ мы обозначим через $\mathcal{L}_{i j}=L\left(\Lambda_{i}, \Sigma_{i j}\right)$ свободный $\mathbb{Z}$-модуль, состоящий из всех конечных формальных линейных комбинаций

$$
\tau=\sum_{\alpha} a_{\alpha}\left(\Lambda_{i} g_{\alpha}\right)
$$

с целочисленными коэффициентами $a_{\alpha}$ символов $\left(\Lambda_{i} g_{\alpha}\right)$ с $g_{\alpha} \in \Sigma_{i j}$, которые взаимно однозначно соответствуют левым классам множества $\Sigma_{i j}$ по группе $\Lambda_{i}$, и через $\mathcal{D}_{i j}$ обозначим подмодуль модуля $\mathcal{L}_{i j}$, состоящий из всех элементов, инвариантных относительно естественного правого умножения на любой элемент группы $\Lambda_{j}$,

$$
\Lambda_{j} \ni \lambda: \tau \mapsto \tau \lambda=\sum_{\alpha} a_{\alpha}\left(\Lambda_{i} g_{\alpha} \lambda\right)
$$

т.е.

$$
\mathcal{D}_{i j}=\left\{\tau \in \mathcal{L}_{i j} \mid \tau \lambda=\tau \text { для всех } \lambda \in \Lambda_{j}\right\} .
$$

Легко видеть, что в качестве базиса подмодуля $\mathcal{D}_{i j}$ можно взять различные элементы вида

$$
\tau(g)=\sum_{g_{\alpha} \in \Lambda_{i} \backslash \Lambda_{i} g \Lambda_{j}}\left(\Lambda_{i} g_{\alpha}\right) \quad\left(g \in \Sigma_{i j}\right),
$$

которые находятся во взаимно однозначном соответствии с различными двойными классами $\Lambda_{i} g \Lambda_{j}$, содержащимися в $\Sigma_{i j}$. Для данных элементов

$$
\tau=\sum_{\alpha} a_{\alpha}\left(\Lambda_{i} g_{\alpha}\right) \in \mathcal{D}_{i j} \quad \text { и } \quad \tau^{\prime}=\sum_{\beta} b_{\beta}\left(\Lambda_{j} g_{\beta}^{\prime}\right) \in \mathcal{D}_{j k},
$$

где $i, j, k=1, \ldots, h$, определим произведение $\tau \tau^{\prime}$, полагая

$$
\tau \tau^{\prime}=\sum_{\alpha, \beta} a_{\alpha} b_{\beta}\left(\Lambda_{i} g_{\alpha} g_{\beta}^{\prime}\right)
$$

Нетрудно видеть, что это произведение не зависит от выбора представителей $g_{\alpha}$ и $g_{\beta}^{\prime}$ в соответствующих левых классах, принадлежит пространству $\mathcal{D}_{i k}$ и определяет билинейное спаривание

$$
\mathcal{D}_{i j} \times \mathcal{D}_{j k} \mapsto \mathcal{D}_{i j} \mathcal{D}_{j k} \subset \mathcal{D}_{i k} .
$$

Наконец, мы обозначим через

$$
\mathcal{D}=\mathcal{D}\langle\mathcal{S}\rangle=D\left(\Lambda_{1}, \ldots, \Lambda_{h} ; \Sigma_{11}, \Sigma_{12}, \ldots, \Sigma_{h h}\right)
$$

$\mathbb{Z}$-модуль, состоящий из всех $(h \times h)$-матриц вида

$$
\boldsymbol{\tau}=\left(\begin{array}{ccc}
\tau_{11} & \ldots & \tau_{1 h} \\
\vdots & \ddots & \vdots \\
\tau_{h 1} & \ldots & \tau_{h h}
\end{array}\right)
$$


где $\tau_{i j} \in \mathcal{D}_{i j}$, с естественными операциями сложения матриц и умножения матриц на скаляры. Ясно, что в качестве базиса пространства $\mathcal{D}$ можно взять множество матриц вида

$$
\boldsymbol{\tau}\left[\left(g_{i j}\right)\right]=\left(\begin{array}{ccc}
\tau\left(g_{11}\right) & \ldots & \tau\left(g_{1 h}\right) \\
\vdots & \ddots & \vdots \\
\tau\left(g_{h 1}\right) & \ldots & \tau\left(g_{h h}\right)
\end{array}\right)
$$

где $g_{i j} \in \Sigma_{i j}$ и $\tau\left(g_{i j}\right) \in \mathcal{D}_{i j}$ обозначают элементы вида (2.5). Если мы теперь определим умножение в $\mathcal{D}$ по обычному правилу умножения матриц,

$$
\boldsymbol{\tau} \boldsymbol{\tau}^{\prime}=\left(\tau_{i j}\right)\left(\tau_{j k}^{\prime}\right)=\left(\sum_{j=1}^{h} \tau_{i j} \tau_{j k}^{\prime}\right)
$$

где произведения $\tau_{i j} \tau_{j k}^{\prime}$ понимаются в смысле формул (2.6), мы, очевидно, получим ассоциативное кольцо, которое будем называть (матричным) кольцом Гекке-Шимуры (НS-кольцом) или кольцом двойных классов системы $\mathcal{S}=$ $\left(\Lambda_{1}, \ldots, \Lambda_{h} ; \Sigma_{11}, \Sigma_{12}, \ldots, \Sigma_{h h}\right)($ над $\mathbb{Z})$. HS-кольцо $\mathcal{D}\langle\mathcal{S}\rangle$ называется ручным, если система $\mathcal{S}$ ручная. Отметим, что кольцо скаляров $\mathbb{Z}$ в определении колец Гекке-Шимуры может быть заменено на произвольное коммутативное и ассоциативное кольцо $\mathbb{A}$ с единичным элементом, что приводит к кольцам ГеккеШимуры над $\mathbb{A}$.

Обратимся теперь к представлениям квадратичных форм квадратичными формами. Квадратичная форма

$$
\mathbf{q}(X)=\frac{1}{2}^{t} X Q X \quad\left({ }^{t} X=\left(x_{1}, \ldots, x_{m}\right)\right)
$$

от $m$ переменных с матрицей $Q$ называется целочисленной, если ее матрица $Q$ содержится в множестве

$$
\mathbb{E}_{m}=\left\{Q=\left(Q_{i j}\right) \in \mathbb{Z}_{m}^{m} \mid Q_{i j}=Q_{j i}, Q_{i i} \in 2 \mathbb{Z}(i, j=1, \ldots, m)\right\}
$$

четных матриц порядка $m$. Форма является неособой, если $\operatorname{det} \mathbf{q}=\operatorname{det} Q \neq 0$. Говоря о целочисленных квадратичных формах, мы будем, главным образом, использовать эквивалентный язык четных матриц. Читатель может легко перевести соответствующие определения и утверждения на язык квадратичных форм.

Для двух матриц $Q$ и $Q^{\prime}$, содержащихся в $\mathbb{E}_{m}$, мы будем обозначать через

$$
R^{+}\left(Q, Q^{\prime}\right)=\left\{D \in \mathbb{Z}_{m}^{m} \mid Q[D]=Q^{\prime}, \operatorname{det} D>0\right\}
$$

множество всех собственных иелочисленных представлений матрицы $Q^{\prime}$ матрицей $Q$. Две неособые матрицы $Q$ и $Q^{\prime}$ из $\mathbb{E}_{m}$ называют собственно подобными и пишут

$$
Q \simeq{ }^{+} Q^{\prime}
$$


если $\operatorname{det} Q=\operatorname{det} Q^{\prime}$ и множество $R^{+}\left(Q, \mu Q^{\prime}\right)$ непусто для некоторого целого положительного числа $\mu$, взаимно простого с $\operatorname{det} Q$. В этом случае любая матрица $D \in R^{+}\left(Q, \mu Q^{\prime}\right)$ называется (собственным) подобием матрицы $Q$ в матрииу $Q^{\prime}$ с мультипликатором $\mu=\mu(D)$. Мы будем обозначать через

$$
S^{+}\left(Q, Q^{\prime}\right)=\bigcup_{\mu \in \mathbb{N}, \operatorname{gcd}(\mu, \operatorname{det} Q)=1} R^{+}\left(Q, \mu Q^{\prime}\right), \quad \text { где } \operatorname{det} Q=\operatorname{det} Q^{\prime},
$$

множество всех (собственных) подобий матрицы $Q$ в матрицу $Q^{\prime}$.

Лемма 2.2. Пусть $Q$ и $Q^{\prime}$ - две неособъе матрицы из $\mathbb{E}_{m}$ с равными определителями. Тогда отображение

$$
S^{+}\left(Q, Q^{\prime}\right) \ni D \mapsto D^{*}=\mu(D) D^{-1}
$$

является биекцией множества $S^{+}\left(Q, Q^{\prime}\right)$ на множество $S^{+}\left(Q^{\prime}, Q\right)$; эта биекция не меняет мультипликаторов и удовлетворяет условию $\left(D^{*}\right)^{*}=D$. Кроме того, если $D \in S^{+}\left(Q, Q^{\prime}\right)$ и $D_{1} \in S^{+}\left(Q^{\prime}, Q^{\prime \prime}\right)$, где $Q, Q^{\prime}$ и $Q^{\prime \prime}$ - четные матрицы одинаковых порядков с равными ненулевыми определителями, mo $D D_{1} \in S^{+}\left(Q, Q^{\prime \prime}\right) u\left(D D_{1}\right)^{*}=D_{1}^{*} D^{*}$.

ДокАЗАТЕЛЬСтво. Если $D \in S^{+}\left(Q, Q^{\prime}\right)$, то матрица $D^{*}=\mu(D) D^{-1}$, очевидно, удовлетворяет соотношениям ${ }^{t} D^{*} Q^{\prime} D^{*}=\mu(D) Q$ и $D^{*}=\left(Q^{\prime}\right)^{-1 t} D Q$. Отсюда следует, что матрица $D^{*}$ является целочисленной, поскольку целочисленны ее произведения на два взаимно простых числа $\operatorname{det} D=\mu^{m / 2}$ и $\operatorname{det} Q^{\prime}=\operatorname{det} Q$, она содержится в множестве $S^{+}\left(Q^{\prime}, Q\right)$ и имеет тот же мультипликатор, что и матрица $D$. Остальное очевидно. Лемма 2.2 доказана.

Отношение собственного подобия является, очевидно, рефлексивным и транзитивным. Кроме того, по лемме 2.2 это отношение симметрично. Отсюда следует, что множество всех неособых четных матриц данного порядка является объединением непересекающихся классов собственного подобия

$$
\langle Q\rangle^{+}=\left\{Q^{\prime} \in \mathbb{E}_{m} \mid Q^{\prime} \simeq^{+} Q\right\}
$$

Далее, напомним, что две четные матрицы $Q$ и $Q^{\prime}$ одинакового порядка $m$ называют собственно эквивалентными, $Q \cong{ }^{+} Q^{\prime}$, если

$$
Q^{\prime}=Q[U] \text { с некоторой матрицей } U \in \Lambda_{+}^{m}=S L_{m}(\mathbb{Z}) \text {. }
$$

Квадратичные формы $\mathbf{q}$ и $\mathbf{q}^{\prime}$ с собственно эквивалентными матрицами называются собственно эквивалентными, $\mathbf{q} \cong{ }^{+} \mathbf{q}^{\prime}$. Множество всех матриц $Q^{\prime}$ (соответственно квадратичных форм $\mathbf{q}^{\prime}$ ), собственно эквивалентных данной матрице $Q$ (соответственно форме q), называется (собственным) классом эквивалентности матрицы $Q$ (соответственно формы q) и обозначается через $\{Q\}^{+}$ (соответственно $\{\mathbf{q}\}^{+}$). Таким образом,

$$
\{Q\}^{+}=\left\{Q^{\prime}=Q[U] \mid U \in \Lambda_{+}^{m}\right\} .
$$

Основные характеристики целочисленной квадратичной формы q с матрицей $Q$, такие как сигнатура матрицы $Q$ (т.е. число положительных 
и отрицательных квадратов при вещественной диагонализации формы q), определитель $d=\operatorname{det} Q$, делитель ненулевой матрицы $Q$ (т.е. наибольшее целое положительное число $\delta$ такое, что матрица $\delta^{-1} Q$ является четной) и cтупень неособой $Q$ (т.е. наименьшее целое положительное число $q$ такое, что матрица $q Q^{-1}$ четна) зависят только от класса эквивалентности (2.14) матрицы $Q$. Согласно теории приведения целочисленных квадратичных форм (см., например, [8; гл. 9]), множество всех четных матриц фиксированного порядка и фиксированного ненулевого определителя является объединением конечного числа классов целочисленно собственно эквивалентных матриц. В частности, каждый собственный класс подобия неособых четных матриц является конечным объединением (непересекающихся) классов собственной эквивалентности,

$$
\langle Q\rangle^{+}=\bigcup_{i=1}^{h^{+}\langle Q\rangle}\left\{Q_{i}\right\}^{+}
$$

Число $h=h^{+}\langle Q\rangle=h^{+}\langle\mathbf{q}\rangle$ мы будем называть числом классов матрицы $Q$. Поскольку ниже мы рассматриваем только собственные подобия, эквивалентности, классы и числа классов, то прилагательное "собственный", так же как и соответствующий индекс “+”, будет, как правило, опускаться после первого упоминания.

Пусть $Q$ - неособая матрица из $\mathbb{E}_{m}$. Мы фиксируем некоторое множество представителей $Q_{1}, \ldots, Q_{h}$ из классов, удовлетворяющих разложению (2.15). Для любой такой системы мы определим подгруппы $\mathbf{E}_{1}, \ldots, \mathbf{E}_{h}$ группы $G=$ $G L_{m}(\mathbb{Q})$, беря в качестве $\mathbf{E}_{i}$ группу

$$
\mathbf{E}_{i}=E^{+}\left(Q_{i}\right)=R^{+}\left(Q_{i}, Q_{i}\right)
$$

(собственных) единии, матрицы $Q_{i}$, и определим подмножества $\mathbf{A}_{i j}$ группы $G$ для $i, j=1, \ldots, h$, полагая $\mathbf{A}_{i j}$ равным множеству

$$
\mathbf{A}_{i j}=\bigcup_{\mu=1}^{\infty} \mathbf{A}_{i j}(\mu), \quad \text { əде } \quad \mathbf{A}_{i j}(\mu)=R^{+}\left(Q_{i}, \mu Q_{j}\right),
$$

(собственных) автоморфов матрицы $Q_{i}$ в $Q_{j}$. В отличие от подобий, мультипликатором автоморфа может быть произвольное целое положительное число.

Мы собираемся определить кольцо Гекке-Шимуры (2.7) системы

$$
\mathcal{S}\langle Q\rangle=\left(\mathbf{E}_{1}, \ldots, \mathbf{E}_{h} ; \mathbf{A}_{11}, \mathbf{A}_{12}, \ldots, \mathbf{A}_{h h}\right),
$$

но сначала необходимо проверить, является ли эта система $h s$-системой. Непосредственно из определений следует, что группы $\Lambda_{i}=\mathbf{E}_{i}$ и множества $\Sigma_{i j}=\mathbf{A}_{i j}$ удовлетворяют условиям (1) и (2) определения $h s$-систем. Для проверки условия (3) мы докажем следующую простую лемму.

Лемма 2.3. Для каждой неособой матрици $D \in \mathbb{Z}_{m}^{m}$ пересечение левого класса $\Lambda D$ по модулю группы $\Lambda=\Lambda_{+}=S L_{m}(\mathbb{Z})$ с множеством $\mathbf{A}_{i j}$ либо пусто, либо состоит из единственного левого класса $\mathbf{E}_{i} D^{\prime}$ множества $\mathbf{A}_{i j}$ по модулю $\mathbf{E}_{i}$. 
ДоказАтельство. Действительно, если $D^{\prime}, D^{\prime \prime} \in \mathbf{A}_{i j} \cap \Lambda D$, то $Q_{i}\left[D^{\prime}\right]=$ $\mu^{\prime} Q_{j}, Q_{i}\left[D^{\prime \prime}\right]=\mu^{\prime \prime} Q_{j}$ и $D^{\prime \prime}=\lambda D^{\prime}$ с $\lambda \in \Lambda$, откуда

$$
Q_{i}[\lambda]=Q_{i}\left[D^{\prime \prime}\left(D^{\prime}\right)^{-1}\right]=\mu^{\prime \prime} Q_{j}\left[\left(D^{\prime}\right)^{-1}\right]=\mu^{\prime \prime} / \mu^{\prime} Q_{i}
$$

и, таким образом, $\mu^{\prime \prime}=\mu^{\prime}, \lambda \in \mathbf{E}_{i}$ и $D^{\prime \prime} \in \mathbf{E}_{i} D^{\prime}$. Лемма 2.3 доказана.

Обратимся теперь к условию (3). Пусть $D \in \mathbf{A}_{i j}$, и пусть

$$
\mathbf{E}_{i} D \mathbf{E}_{j}=\bigcup_{\alpha} \mathbf{E}_{i} D_{\alpha}
$$

- разбиение двойного класса в объединение непересекающихся левых классов. Тогда

$$
\bigcup_{\alpha} \mathbf{E}_{i} D_{\alpha} \subset \Lambda D \Lambda=\bigcup_{\beta} \Lambda D_{\beta}^{\prime},
$$

и по лемме 2.3 каждый левый класс $\Lambda D_{\beta}^{\prime}$ содержит не более одного левого класса $\mathbf{E}_{i} D_{\alpha}$. Но объединение справа является конечным (см., например, [9; §3.2]), так что конечно и объединение слева. Таким образом, мы можем определить кольцо (2.7) двойных классов системы $\mathcal{S}\langle Q\rangle$,

$$
\mathcal{H}\langle Q\rangle=\mathcal{H}\left(Q_{1}, \ldots, Q_{h}\right)=D\left(\mathbf{E}_{1}, \ldots, \mathbf{E}_{h} ; \mathbf{A}_{11}, \mathbf{A}_{12}, \ldots, \mathbf{A}_{h h}\right),
$$

обобщающее кольцо (1.2), которое мы будем называть кольцом Гекке-Шимуры или кольцом классов автоморфов матрицы $Q$ (над $\mathbb{Z}$ ). В последующем изложении мы фиксируем матрицу $Q$ и все обозначения, относящиеся к определению кольца $\mathcal{H}\langle Q\rangle$, включая некоторую систему $Q_{1}, \ldots, Q_{h}$ представителей из классов целочисленной эквивалентности, содержащихся в классе подобия (2.15).

Элементы кольца $\mathcal{H}\langle Q\rangle$ вида (2.8),

$$
\boldsymbol{\tau}\left[\left(D_{i j}\right)\right]=\left(\tau\left(D_{i j}\right)\right) \quad\left(D_{i j} \in \mathbf{A}_{i j}, \tau\left(D_{i j}\right)=\sum_{D_{i j}^{\alpha} \in \mathbf{E}_{i} \backslash \mathbf{E}_{i} D_{i j} \mathbf{E}_{j}}\left(\mathbf{E}_{i} D_{i j}^{\alpha}\right)\right),
$$

образуют базис этого кольца над $\mathbb{Z}$. Элементы $(2.19)$, где все матрицы $D_{i j}$ содержатся в соответствующих подмножествам подобий

$$
\mathbf{S}_{i j}=\bigcup_{\mu \geqslant 1, \operatorname{gcd}(\mu, q)=1} \mathbf{A}_{i j}(\mu) \subset \mathbf{A}_{i j},
$$

и их линейные комбинации с целочисленными коэффициентами мы будем называть регулярными элементами кольца $\mathcal{H}\langle Q\rangle$. Подмножество $\mathcal{H}_{r}\langle Q\rangle$ всех регулярных элементов образует, очевидно, подкольцо кольца $\mathcal{H}\langle Q\rangle$, регулярное подкольио кольца $\mathcal{H}\langle Q\rangle$ или кольцо классов подобия матрицы $Q$, которое само может быть интерпретировано как кольцо двойных классов:

$$
\mathcal{H}_{r}\langle Q\rangle=D\left(\mathbf{E}_{1}, \ldots, \mathbf{E}_{h} ; \mathbf{S}_{11}, \mathbf{S}_{12}, \ldots, \mathbf{S}_{h h}\right) \subset \mathcal{H}\langle Q\rangle .
$$

Для элемента

$$
\boldsymbol{\tau}=\sum_{\alpha} a_{\alpha}\left(\tau\left(D_{i j}^{\alpha}\right)\right) \in \mathcal{H}_{r}\langle Q\rangle
$$

мы положим

$$
\boldsymbol{\tau}^{*}=\sum_{\alpha} a_{\alpha}{ }^{t}\left(\tau^{*}\left(D_{i j}^{\alpha}\right)\right), \quad \text { где } \quad \tau^{*}(D)=\tau\left(D^{*}\right)=\tau\left(\mu(D) D^{-1}\right) .
$$


ЛЕмма 2.4. Отображение $\boldsymbol{\tau} \mapsto \boldsymbol{\tau}^{*}$ является линейным антиавтоморфизмом порядка 2 колъиа классов подобия $\mathcal{H}_{r}\langle Q\rangle$.

ДокАзАтЕльство. По лемме 2.2 отображение $\boldsymbol{\tau} \mapsto \boldsymbol{\tau}^{*}$ является линейным отображением кольца $\boldsymbol{\mathcal { H }}_{r}\langle Q\rangle$ в себя и удовлетворяет соотношению $\left(\boldsymbol{\tau}^{*}\right)^{*}=\boldsymbol{\tau}$. В частности, это отображение взаимно однозначно.

Остается проверить, что отображение $\boldsymbol{\tau} \mapsto \boldsymbol{\tau}^{*}$ является мультипликативным антигомоморфизмом, т.е. удовлетворяет соотношениям

$$
\left(\boldsymbol{\tau} \boldsymbol{\tau}_{1}\right)^{*}=\boldsymbol{\tau}_{1}^{*} \boldsymbol{\tau}^{*} \quad \text { для } \quad \boldsymbol{\tau}, \boldsymbol{\tau}_{1} \in \mathcal{H}_{r}\langle Q\rangle .
$$

Эти соотношения достаточно проверить для базисных элементов (2.19) кольца $\mathcal{H}_{r}\langle Q\rangle$. Прежде всего отметим, что с помощью вполне элементарных, но довольно утомительных вычислений, основанных на технике, использованной в доказательстве предложения 3.1.7 книги [9], можно проверить, что

$$
\left(\tau(D) \tau\left(D^{\prime}\right)\right)^{*}=\tau^{*}\left(D^{\prime}\right) \tau^{*}(D) \text { для всех } D \in \mathbf{S}_{i k}, D^{\prime} \in \mathbf{S}_{k j}(i, j, k=1, \ldots, h),
$$

где отображение * слева по линейности продолжено на целочисленные линейные комбинации двойных классов $\tau\left(D^{\prime \prime}\right)$ с $D^{\prime \prime} \in \mathbf{S}_{i j}$. Тогда, с одной стороны, мы имеем

$$
\begin{aligned}
\left(\left(\tau\left(D_{i k}\right)\right)\left(\tau\left(D_{k j}^{\prime}\right)\right)\right)_{i j}^{*} & =\left(\sum_{k} \tau\left(D_{i k}\right) \tau\left(D_{k j}^{\prime}\right)\right)_{i j}^{*}=\left(\sum_{k} \tau^{*}\left(D_{k j}^{\prime}\right) \tau^{*}\left(D_{i k}\right)\right)_{j i} \\
& =\left(\sum_{k} \tau^{*}\left(D_{k i}^{\prime}\right) \tau^{*}\left(D_{j k}\right)\right)_{i j} .
\end{aligned}
$$

А с другой стороны, получаем

$$
\left(\left(\tau\left(D_{i k}^{\prime}\right)\right)^{*}\left(\tau\left(D_{k j}\right)\right)^{*}\right)_{i j}=\left(\left(\tau^{*}\left(D_{k i}^{\prime}\right)\right)\left(\tau^{*}\left(D_{j k}\right)\right)\right)_{i j}=\left(\sum_{k} \tau^{*}\left(D_{k i}^{\prime}\right) \tau^{*}\left(D_{j k}\right)\right)_{i j}
$$

Сравнение этих выражений доказывает соотношения (2.23) для базисных элементов. Лемма 2.4 доказана.

Вообще говоря, кольцо $\mathcal{H}=\mathcal{H}\left(Q_{1}, \ldots, Q_{h}\right)$ некоммутативно, однако при определенных условиях элементы этого кольца удовлетворяют важным коммутационным соотношениям, аналогичным соотношениям (1.4) в случае $h=1$. Чтобы сформулировать эти соотношения, мы введем некоторые обозначения. Согласно теории элементарных делителей для группы $\Lambda=G L_{m}(\mathbb{Z})$ (см., например [9; лемма 3.2.2]), каждый двойной класс $\Lambda D \Lambda$ неособой матрицы $D \in \mathbb{Z}_{m}^{m}$ содержит единственного диагонального представителя вида

$$
\operatorname{ed}(D)=\operatorname{diag}\left(d_{1}, \ldots, d_{m}\right) \quad \text { с } \quad d_{i} \in \mathbb{N} \quad \text { и } \quad d_{i} \mid d_{i+1} .
$$

Если $\operatorname{det} D>0$, то то же, очевидно, справедливо и для двойного класса $\Lambda_{+} D \Lambda_{+}$ по группе $\Lambda_{+}=S L_{m}(\mathbb{Z})$. Диагональная матрица ed $(D)$ называется матрицей элементарных делителей матрицы $D$, а числа $d_{i}=d_{i}(D)$ ее элементарными делителями. Элементарные делители удовлетворяют соотношениям

$$
d_{i}(D) d_{i}\left(D^{\prime}\right)=d_{i}\left(D D^{\prime}\right) \text { для } i=1, \ldots, m, \text { если } \operatorname{gcd}\left(\operatorname{det}(D), \operatorname{det}\left(D^{\prime}\right)\right)=1,
$$


и соотношениям

$$
d_{1}(D) \cdots d_{m}(D)=|\operatorname{det} D| .
$$

Для каждой матрицы элементарных делителей

$$
D=\operatorname{ed}(D)=\operatorname{diag}\left(d_{1}, \ldots, d_{m}\right)
$$

Мы определим элемент кольца $\mathcal{H}$ вида

$$
\boldsymbol{\tau}(D)=\boldsymbol{\tau}\left[d_{1}, \ldots, d_{m}\right]=\left(\tau_{i j}\left(d_{1}, \ldots, d_{m}\right)\right),
$$

где для $i, j=1, \ldots, h$ мы полагаем

$$
\tau_{i j}(D)=\tau_{i j}\left[d_{1}, \ldots, d_{m}\right]= \begin{cases}\sum_{D^{\prime} \in \mathbf{E}_{i} \backslash \mathbf{A}_{i j} \cap \Lambda_{+} D \Lambda_{+}}\left(\mathbf{E}_{i} D^{\prime}\right), & \text { если } \mathbf{A}_{i j} \cap \Lambda_{+} D \Lambda_{+} \neq \varnothing, \\ 0, & \text { если } \mathbf{A}_{i j} \cap \Lambda_{+} D \Lambda_{+}=\varnothing,\end{cases}
$$

и $\mathbf{A}_{i j}$ - множества автоморфов (2.17). Кроме того, для целого положительного числа $\mu$ мы введем сумму элементов (2.28) вида

$$
\boldsymbol{\tau}(\mu)=\sum_{\substack{d_{i} \in \mathbb{N}, d_{i} \mid d_{i+1} \\ d_{1} \cdots d_{m}=\mu^{m / 2}}} \tau\left[d_{1}, \ldots, d_{m}\right]=\left(\tau_{i j}(\mu)\right),
$$

аналогичную элементу (1.3), где

$$
\tau_{i j}(\mu)=\sum_{D^{\prime} \in \mathbf{E}_{i} \backslash \mathbf{A}_{i j}(\mu)}\left(\mathbf{E}_{i} D^{\prime}\right) .
$$

Наконец, будет удобно определить для целых положительных $d$ "скалярные" элементы кольца $\mathcal{H}$ вида

$$
[d]=[d]_{m}=\boldsymbol{\tau}[\underbrace{d, \ldots, d}_{m}]=\operatorname{diag}\left(\left(\mathbf{E}_{1}\left(d \cdot \mathbf{1}_{m}\right)\right), \ldots,\left(\mathbf{E}_{h}\left(d \cdot \mathbf{1}_{m}\right)\right)\right) .
$$

Лемма 2.5. Элементарные делители каждого подобия $D \in \mathbf{S}_{i j}$ удовлетворяют соотношениям

$$
\operatorname{ed}(D)=\operatorname{ed}\left(\mu(D) D^{-1}\right) \Leftrightarrow d_{k}(D) d_{m-k+1}(D)=\mu(D) \quad(k=1, \ldots, m),
$$

т.е. соответствующие элементы (2.28) удовлетворяют условию

$$
\boldsymbol{\tau}(D)^{*}=\boldsymbol{\tau}(D) \quad\left(D \in \mathbf{S}_{i j}, i, j=1, \ldots, h\right)
$$

в частности,

$$
\boldsymbol{\tau}(\mu)^{*}=\boldsymbol{\tau}(\mu), \quad \text { если } \operatorname{gcd}(\mu, \operatorname{det} Q)=1 .
$$

ДокАЗАтельство. Так как $\operatorname{det} Q_{i}=\operatorname{det} Q_{j}=\operatorname{det} Q$, то из соотношения $Q_{i}[D]=\mu(D) Q_{j}$ следует, что $\operatorname{det} D=\mu(D)^{m / 2}$ и ${ }^{t} D Q_{i}=Q_{j} \mu(D) D^{-1}$. Так как $\operatorname{gcd}(\mu(D), \operatorname{det} Q)=1$, то из последнего соотношения, согласно (2.26), следует соотношение $\operatorname{ed}(D) \operatorname{ed}(Q)=\operatorname{ed}(Q) \operatorname{ed}\left(\mu(D) D^{-1}\right)$. Остальное следует непосредственно из определений. Лемма 2.5 доказана. 
ТеОрема 2.6. Пусть $Q$ - неособая четная матрица порядка $m$, и пусть $\boldsymbol{\tau}(D)=\boldsymbol{\tau}\left[d_{1}, \ldots, d_{m}\right], \boldsymbol{\tau}\left(D^{\prime}\right)=\tau\left[d_{1}^{\prime}, \ldots, d_{m}^{\prime}\right]-$ два ненулевъх элемента вида (2.28). Предположим, что элементарные делители матрии, $D$ и $D^{\prime}$ удовлетворяют условиям

$$
\begin{gathered}
\operatorname{gcd}\left(d_{m} / d_{1}, d_{m}^{\prime} / d_{1}^{\prime}\right)=1 \quad u \\
\operatorname{gcd}\left(d_{m} / d_{1}, \operatorname{det} Q\right)=1 \quad \text { или } \quad \operatorname{gcd}\left(d_{m}^{\prime} / d_{1}^{\prime}, \operatorname{det} Q\right)=1 .
\end{gathered}
$$

Тогда в кольце $\mathcal{H}(Q)$ имеют место следующие соотношения:

$$
\boldsymbol{\tau}(D) \boldsymbol{\tau}\left(D^{\prime}\right)=\boldsymbol{\tau}\left(D D^{\prime}\right)=\boldsymbol{\tau}\left(D^{\prime}\right) \boldsymbol{\tau}(D)
$$

В частности, для каждого элемента вида (2.28) и каждого элемента (2.30) выполняются соотношения

$$
[d] \boldsymbol{\tau}(D)=\boldsymbol{\tau}(D)[d]=\boldsymbol{\tau}(d D) .
$$

ДокАЗАТЕЛьство. Мы следуем схеме доказательства, использованной в работе [7; теорема 2.2], с необходимыми изменениями. Отметим, прежде всего, что частный случай (2.34) немедленно и прямо следует из определений. Поэтому соотношения (2.33) достаточно доказать, предполагая также, что $d_{1}=$ $d_{1}^{\prime}=1$. В этом случае условия (2.32), очевидно, могут быть записаны в виде

$$
\begin{gathered}
\operatorname{gcd}\left(\operatorname{det} D, \operatorname{det} D^{\prime}\right)=1 \quad \text { и } \\
\operatorname{gcd}(\operatorname{det} D, \operatorname{det} Q)=1 \quad \text { или } \operatorname{gcd}\left(\operatorname{det} D^{\prime}, \operatorname{det} Q\right)=1,
\end{gathered}
$$

что мы и будем предполагать ниже. Для доказательства первого из соотношений (2.33) достаточно проверить, что

$$
\sum_{k} \tau_{i k}(D) \tau_{k j}\left(D^{\prime}\right)=\tau_{i j}\left(D D^{\prime}\right) \quad(i, j=1, \ldots, h)
$$

Пусть

$$
\tau_{i k}(D)=\sum_{\alpha}\left(\mathbf{E}_{i} A_{i k}^{\alpha}\right), \quad \tau_{k j}\left(D^{\prime}\right)=\sum_{\beta}\left(\mathbf{E}_{k} B_{k j}^{\beta}\right)
$$

и

$$
\tau_{i j}\left(D D^{\prime}\right)=\sum_{\gamma}\left(\mathbf{E}_{i} C_{i j}^{\gamma}\right)
$$

Тогда мы должны показать, что в качестве множества представителей $C_{i j}^{\gamma}$ из классов $\mathbf{E}_{i} \backslash \mathbf{A}_{i j} \cap \Lambda_{+} D D^{\prime} \Lambda_{+}$можно взять множество всех произведений вида $A_{i k}^{\alpha} B_{k j}^{\beta}$. Так как определители $\operatorname{det} D$ и $\operatorname{det} D^{\prime}$ взаимно просты, то по аналогии с [9; предложение 3.2.5] можно легко проверить, что следующие соотношения выполняются в кольце вида (2.7) двойных классов $D(\Lambda, \Sigma)$ полугруппы $\Sigma=$ $\left\{M \in \mathbb{Z}_{m}^{m} \mid \operatorname{det} M>0\right\}$ по группе $\Lambda=\Lambda_{+}^{m}$ :

$$
\sum_{A^{\prime} \in \Lambda \backslash \Lambda D \Lambda}\left(\Lambda A^{\prime}\right) \cdot \sum_{B^{\prime} \in \Lambda \backslash \Lambda D^{\prime} \Lambda}\left(\Lambda B^{\prime}\right)=\sum_{A^{\prime}, B^{\prime}}\left(\Lambda A^{\prime} B^{\prime}\right)=\sum_{C^{\prime} \in \Lambda \backslash \Lambda D D^{\prime} \Lambda}\left(\Lambda C^{\prime}\right) .
$$

Из этих соотношений и леммы 2.2 следует, что все произведения $A_{i k}^{\alpha} B_{j k}^{\beta}$ содержатся в двойном классе $\Lambda D D^{\prime} \Lambda$ и принадлежат различным левым классам по модулю $\Lambda$, содержащимся в этом двойном классе. В частности, они 
принадлежат различным левым классам по подгруппе $\mathbf{E}_{i} \subset \Lambda$. Пусть теперь $C^{\prime} \in \mathbf{E}_{i} \backslash \mathbf{A}_{i j} \cap \Lambda D D^{\prime} \Lambda$ - произвольный представитель. В силу (2.35) матрицу $C^{\prime}$ можно записать в виде

$$
C^{\prime}=A^{\prime} B^{\prime}, \quad \text { где } \quad A^{\prime} \in \Lambda D \Lambda, \quad B^{\prime} \in \Lambda D^{\prime} \Lambda .
$$

Так как $C^{\prime} \in \mathbf{A}_{i j}$, то $Q_{i}\left[A^{\prime} B^{\prime}\right]=\mu \mu^{\prime} Q_{j}$, и поэтому

$$
\mu^{-1} Q_{i}\left[A^{\prime}\right]=\mu^{\prime} Q_{j}\left[\left(B^{\prime}\right)^{-1}\right],
$$

где $\mu=\mu(D)$ и $\mu^{\prime}=\mu\left(D^{\prime}\right)$. Знаменатели коэффициентов рациональной симметрической матрицы слева в (2.36) являются произведениями простых чисел, делящих число $\mu=(\operatorname{det} D)^{2 / m}$, в то время как знаменатели коэффициентов матрицы справа являются произведениями простых чисел, делящих число $\operatorname{det} B^{\prime}=\operatorname{det} D^{\prime}$. Поскольку числа $\operatorname{det} D$ и $\operatorname{det} D^{\prime}$ взаимно просты, обе матрицы в соотношении (2.36) являются целочисленными. Кроме того, поскольку по меньшей мере одно из чисел $\operatorname{det} D, \operatorname{det} D^{\prime}$ нечетно, обе матрицы являются четными. Так как по меньшей мере одно из чисел $\operatorname{det} D, \operatorname{det} D^{\prime}$, скажем, $\operatorname{det} D=\operatorname{det} A^{\prime}$, взаимно просто с $\operatorname{det} Q=\operatorname{det} Q_{i}$, то четная матрица $\mu^{-1} Q_{i}\left[A^{\prime}\right]$ подобна матрице $Q_{i}$ и, таким образом, эквивалентна одной из матриц $Q_{1}, \ldots, Q_{h}$, скажем, матрице $Q_{k}$ :

$$
\mu^{-1} Q_{i}\left[A^{\prime}\right]=Q_{k}[\lambda] \quad \text { c } \quad \lambda \in \Lambda,
$$

так что $Q_{i}\left[A^{\prime} \lambda^{-1}\right]=\mu Q_{k}$. Таким образом, $A^{\prime} \lambda^{-1} \in \mathbf{A}_{i k} \cap \Lambda D \Lambda$, откуда $A^{\prime} \lambda^{-1}=$ $\delta A_{i k}^{\gamma}$ с $\delta \in \mathbf{E}_{i}$. Но тогда поскольку

$$
\mu \mu^{\prime} Q_{j}=Q_{i}\left[A^{\prime} B^{\prime}\right]=Q_{i}\left[A^{\prime} \lambda^{-1} \cdot \lambda B^{\prime}\right]=\mu Q_{k}\left[\lambda B^{\prime}\right],
$$

то $\lambda B^{\prime} \in \mathbf{A}_{k j} \cap \Lambda D^{\prime} \Lambda$, и отсюда $\lambda B^{\prime}=\delta_{1} B_{k j}^{\beta}$ с $\delta_{1} \in \mathbf{E}_{k}$. Тогда

$$
C^{\prime}=A^{\prime} B^{\prime}=\delta A_{i k}^{\gamma} \delta_{1} B_{k j}^{\beta}=\delta \delta^{\prime} A_{i k}^{\alpha} B_{k j}^{\beta},
$$

где $\delta \delta^{\prime} \in \mathbf{E}_{i}$. Это доказывает теорему 2.6.

СлеДСтвиЕ 2.7. Элементы вида (2.29) удовлетворяют соотношениям

$$
\boldsymbol{\tau}(\mu) \boldsymbol{\tau}\left(\mu^{\prime}\right)=\boldsymbol{\tau}\left(\mu \mu^{\prime}\right)=\boldsymbol{\tau}\left(\mu^{\prime}\right) \boldsymbol{\tau}(\mu),
$$

если

$$
\begin{gathered}
\operatorname{gcd}\left(\mu, \mu^{\prime}\right)=1 \quad u \\
\operatorname{gcd}(\mu, \operatorname{det} Q)=1 \quad \text { или } \quad \operatorname{gcd}\left(\mu^{\prime}, \operatorname{det} Q\right)=1 .
\end{gathered}
$$

ДокАзАтЕльство. Суммируя соотношения (2.33) по всем матрицам элементарных делителей вида $D=\operatorname{diag}\left(d_{1}, \ldots, d_{m}\right)$ с $d_{1} \cdots d_{m}=\mu^{m / 2}$ и вида $D^{\prime}=\operatorname{diag}\left(d_{1}^{\prime}, \ldots, d_{m}^{\prime}\right)$ с $d_{1}^{\prime} \cdots d_{m}^{\prime}=\left(\mu^{\prime}\right)^{m / 2}$, мы, очевидно, получаем соотношения (2.37).

На основании следствия 2.7 мы заключаем, что формальный ряд Дирихле с коэффициентами $\boldsymbol{\tau}(1), \boldsymbol{\tau}(2), \ldots$, аналогично разложению (1.5), может быть разложен в формальное эйлерово произведение:

$$
\sum_{\mu=1}^{\infty} \frac{\boldsymbol{\tau}(\mu)}{\mu^{s}}=\left(\sum_{\nu \mid(\operatorname{det} Q)^{\infty}} \frac{\boldsymbol{\tau}(\nu)}{\nu^{s}}\right) \prod_{p \nmid \operatorname{det} Q} \sum_{\delta=0}^{\infty} \frac{\boldsymbol{\tau}\left(p^{\delta}\right)}{p^{\delta s}},
$$


где мы рассматриваем выражение $\mu^{s}$ просто как формальный квазихарактер мультипликативной полугруппы $\mathbb{N}$ и где $\nu$ и $p$ пробегают все положительные целые числа, делящие некоторую степень числа $\operatorname{det} Q$, и простые числа, не делящие число $\operatorname{det} Q$, соответственно. В работе [3] было выдвинуто предположение, что для каждого простого числа $p$, не делящего $\operatorname{det} Q$, формальный степенной ряд с коэффициентами $\boldsymbol{\tau}(1)=[1], \boldsymbol{\tau}(p), \boldsymbol{\tau}\left(p^{2}\right), \ldots$ является (формально) рациональной дробью над кольцом $\mathcal{H}\langle Q\rangle$ со знаменателем степени $2^{k}$ и числителем степени не больше $2^{k}-2$, если порядок $m$ матрицы $Q$ нечетен вида $2 k-1$ или четен вида $2 k$ :

$$
\sum_{\delta=0}^{\infty} \boldsymbol{\tau}\left(p^{\delta}\right) t^{\delta}=R_{p}(t)^{-1} S_{p}(t)
$$

где

$$
R_{p}(t)=[1]+\sum_{1 \leqslant i \leqslant 2^{k}} \rho_{i} t^{i}, \quad S_{p}(t)=[1]+\sum_{1 \leqslant j \leqslant 2 k-2} \sigma_{j} t^{j}
$$

с $\rho_{i}=\rho_{i}(p), \sigma_{j}=\sigma_{j}(p) \in \mathcal{H}\langle Q\rangle$. В этом случае мы будем говорить, что формальный степенной ряд над кольцом $\mathcal{H}\langle Q\rangle$ вида

$$
Z_{p}(t, Q)=Z_{p}(t, \mathbf{q})=R_{p}(t)^{-1}
$$

является локальным дзета-рядом матрицы $Q$. В работе [3] было доказано, что это предположение справедливо для любых четных неособых матриц $Q$ порядков $m=2,3$ и 4 . А именно, для каждого простого числа $p$, не делящего $\operatorname{det} Q$, имеют место следующие формальные тождества:

$$
\begin{aligned}
& \sum_{\delta=0}^{\infty} \boldsymbol{\tau}\left(p^{\delta}\right) t^{\delta}
\end{aligned}
$$

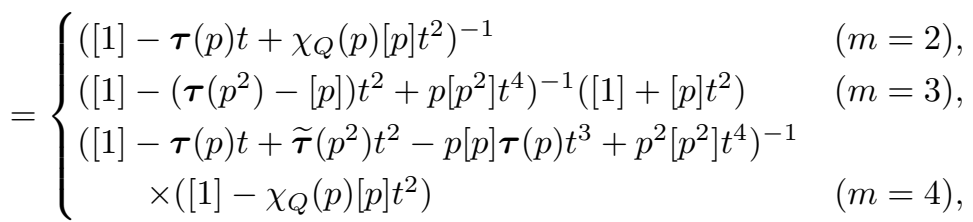

где $\chi_{Q}$ в случае $m=2$ обозначает характер квадратичной формы $\mathbf{q}(X)$ с матрицей $Q$, т.е. (для $p$, не равных 2 и не делящих $\operatorname{det} Q) \chi_{Q}(p)=1$, если сравнение $\mathbf{q}(X) \equiv 0(\bmod p)$ имеет нетривиальное решение, и $\chi_{Q}(p)=-1$ в противном случае, а в случае $m=4$ значение $\chi_{Q}(p)=\left(\frac{\operatorname{det} Q}{p}\right)$ равно символу Лежандра, и где

$$
\widetilde{\boldsymbol{\tau}}\left(p^{2}\right)=\chi_{Q}(p) \boldsymbol{\tau}\left[1, p, p, p^{2}\right]+\left(1+\chi_{Q}(p)\right) p[p]
$$

(см. работы [10] и [3; теоремы 1.1 и 1.3]). Отсюда следует, что в этих случаях локальные дзета-ряды имеют вид

$$
\begin{array}{ll}
Z_{p}(t, Q)=\left([1]-\boldsymbol{\tau}(p) t+\chi_{Q}(p)[p] t^{2}\right)^{-1} & (m=2) ; \\
Z_{p}(t, Q)=\left([1]-\left(\boldsymbol{\tau}\left(p^{2}\right)-[p]\right) t^{2}+p\left[p^{2}\right] t^{4}\right)^{-1} & (m=3) \\
Z_{p}(t, Q)=\left([1]-\boldsymbol{\tau}(p) t+\widetilde{\boldsymbol{\tau}}\left(p^{2}\right) t^{2}-p[p] \boldsymbol{\tau}(p) t^{3}+p^{2}\left[p^{2}\right] t^{4}\right)^{-1} & (m=4) .
\end{array}
$$


Из приведенных формул суммирования следуют новые коммуникационные соотношения в кольцах классов подобия (2.20) четных неособых матриц $Q$ порядков 2,3 и 4 .

ПреДЛОЖЕНИЕ 2.8. Пусть $Q$ - четная неособая матрица порядка $m=2,3$ или 4, и пусть р - простое число, не делящее число $\operatorname{det} Q$. Тогда все элементь вида $\boldsymbol{\tau}\left(p^{\delta}\right) \in \mathcal{H}_{r}\langle Q\rangle$ с $\delta=0,1,2, \ldots$ принадлежат кольиу многочленов над $\mathbb{Z}$ от коммутирующих друг с другом элементов $\boldsymbol{\tau}(p),[p]$, если $m=2$, элементов $\boldsymbol{\tau}\left(p^{2}\right),[p]$, если $m=3$, элементов $\boldsymbol{\tau}(p), \boldsymbol{\tau}\left[1, p, p, p^{2}\right],[p]$, если $m=4$ u $\chi_{Q}(p)=1$, и элементов $\boldsymbol{\tau}\left[1, p, p, p^{2}\right],[p]$, если $m=4$ и $\chi_{Q}(p)=-1$.

ДокАЗАТЕЛЬство. В случаях $m=2$ и $m=3$ утверждение непосредственно следует из формул (2.41) и (2.34). В случае $m=4$ по той же причине достаточно проверить, что элемент $\boldsymbol{\tau}\left[1, p, p, p^{2}\right]$ коммутирует с $\boldsymbol{\tau}(p)$, если $\chi_{Q}(p)=1$, и что $\boldsymbol{\tau}(p)=0$, если $\chi_{Q}(p)=-1$. Последнее следует из $[3 ;(6.13)]$. Если $\chi_{Q}(p)=1$, то по $[3 ;(6.37)]$ получаем соотношение

$$
\boldsymbol{\tau}\left[1, p, p, p^{2}\right] \boldsymbol{\tau}(p)=\boldsymbol{\tau}\left[1, p, p^{2}, p^{3}\right]+(p+1)[p] \boldsymbol{\tau}(p) .
$$

Откуда, используя леммы 2.5 и 2.4 , мы имеем

$$
\boldsymbol{\tau}\left[1, p, p, p^{2}\right] \boldsymbol{\tau}(p)=\left(\boldsymbol{\tau}\left[1, p, p, p^{2}\right] \boldsymbol{\tau}(p)\right)^{*}=\boldsymbol{\tau}(p)^{*} \boldsymbol{\tau}\left[1, p, p, p^{2}\right]^{*}=\boldsymbol{\tau}(p) \boldsymbol{\tau}\left[1, p, p, p^{2}\right] .
$$

Предложение 2.8 доказано.

Предположим теперь, что задано комплексное представление

$$
\mathcal{H}_{r}\langle Q\rangle \ni \boldsymbol{\tau} \mapsto \mid \boldsymbol{\tau}
$$

кольца $\mathcal{H}_{r}\langle Q\rangle$ линейными операторами, и пусть $P$ - некоторый собственный вектор всех операторов $\mid \boldsymbol{\tau}$ вида $\mid \boldsymbol{\tau}(\mu)$ для $\mu$, взаимно простых со ступенью $q$ матрицы $Q$, так что $P \mid \boldsymbol{\tau}(\mu)=\lambda(\boldsymbol{\tau}(\mu)) P$, где $\lambda(\boldsymbol{\tau}(\mu))$ обозначают соответствующие собственные числа. Тогда по аналогии с теорией дзета-функций зигелевых модулярных форм можно рассмотреть степенные ряды

$$
Z_{p}(t, P)=Z_{p}(t, P, Q)=\left(1+\sum_{1 \leqslant i \leqslant 2^{k}} \lambda\left(\rho_{i}\right) t^{i}\right)^{-1}, \quad \text { где } t=p^{-s},
$$

и эйлерово произведение

$$
Z(s, P)=\prod_{p \nmid \operatorname{det} Q} Z_{p}\left(p^{-s}, P\right),
$$

которые естественно называть соответственно локальной и глобальной дзетафункцией колъца $\mathcal{H}_{r}\langle Q\rangle$, отвечающей собственному вектору $P$, и поставить вопрос о свойствах этих дзета-функций. Ниже мы увидим, что в некоторых случаях ортогональные дзета-функции могут быть явно выражены через спинорные дзета-функции подходящих зигелевых модулярных форм. 


\section{§ 3. Представления на гармонических векторах}

В этом параграфе мы определим линейные представления колец классов автоморфов положительно-определенных квадратичных форм на пространствах гармонических векторов и рассмотрим вопрос о существовании собственных векторов для этих представлений.

Сначала мы напомним определение и свойства гармонических многочленов относительно положительно-определенных квадратичных форм. Многочлен $P_{0}=P_{0}(X)$ от $m n$ переменных $x_{i j}$ над $\mathbb{C}$, где $X=\left(x_{i j}\right)$ - матрица из переменных размера $m \times n$, называется гармоническим многочленом порядка $m$, рода $n$ $u$ веса $k$, где $k$ - целое неотрицательное число, если он является гармонической функцией от $m n$ переменных в том смысле, что выполняется условие

$$
\Delta P_{0}=\sum_{i j} \frac{\partial^{2} P_{0}}{\partial x_{i j}^{2}}=0
$$

и имеют место соотношения

$$
P_{0}(X A)=(\operatorname{det} A)^{k} P_{o}(X) \quad \text { для любых матриц } A \in G L_{n}(\mathbb{C}) .
$$

Из определения следует, что для каждого гармонического многочлена $P_{0}(X)$ порядка $m$, рода $n$ и веса $k$ и каждой матрицы $U$ из вещественной ортогональной группы $O_{m}(\mathbb{R})$ порядка $m$ многочлен $P_{0}(U X)$ снова является гармоническим порядка $m$, рода $n$ и веса $k$. В этом смысле определение гармонических многочленов связано с квадратичной формой $\mathbf{q}_{0}=x_{1}^{2}+\cdots+x_{m}^{2}$ с матрицей $Q_{0}=2 \cdot 1_{m}$, группой вещественных автоморфизмов которой является как раз ортогональная группа порядка $m$. Теперь мы определим гармонические многочлены, отвечающие в том же смысле любой вещественной положительноопределенной квадратичной форме (2.9) от $m$ переменных с матрицей $Q$ : так как форма положительно определена, то существует вещественная матрица $S$ такая, что

$$
Q=2^{t} S S
$$

В этом случае мы определим для гармонического многочлена $P_{0}$ порядка $m$, рода $n$ и веса $k$ гармонический многочлен $P=P_{Q}(X)$ рода $n$ и веса $k$ относительно квадратичной формы с матричей $Q$ (или просто относительно $Q$ ) формулой

$$
P(X)=P_{Q}(X)=\left(P_{0} \mid S\right)(X)=P_{0}(S X) .
$$

Это многочлен от элементов матрицы $X$, который на основании условия (3.2) удовлетворяет соотношениям

$$
P(X A)=(\operatorname{det} A)^{k} P(X) \quad \text { для каждой матрицы } A \in G L_{n}(\mathbb{C}) .
$$

Ясно также, что для каждой матрицы

$$
U \in O(Q, R)=\left\{U \in \mathbb{R}_{m}^{m} \mid Q[U]=Q\right\}=S^{-1} O_{m}(\mathbb{R}) S
$$

многочлен

$$
(P \mid U)(X)=P(U X)=P_{0}(S U X)=\left(P_{0} \mid S U S^{-1}\right)(S X)
$$


снова является гармоническим многочленом рода $n$ и веса $k$ относительно $Q$. Множество $\mathcal{P}_{k}^{n}(Q)$ всех гармонических многочленов рода $n$ и веса $k$ относительно $Q$ образует, очевидно, линейное пространство над полем $\mathbb{C}$. Из соотношений (3.4) следует, что каждый из многочленов, содержащихся в пространстве $\mathcal{P}_{k}^{n}(Q)$, является однородным степени $n k$. Таким образом, пространство $\mathcal{P}_{k}^{n}(Q)$ конечномерно. Следующая лемма содержит описание пространств гармонических многочленов рода $n$ и веса $k$ относительно положительно-определенных квадратичных форм.

ПРеДЛОЖЕНИЕ 3.1. Пространство $\mathcal{P}_{k}^{n}(Q)$ гармонических многочленов относительно матрицы $Q$ положительно-определенной квадратичной формы от $m$ переменных линейно порождается над $\mathbb{C}$ многочленами вида

$$
P(X)=\operatorname{det}\left({ }^{t} \Omega Q X\right)^{k},
$$

где $\Omega$ - некоторая матрица из $\mathbb{C}_{n}^{m}$, удовлетворяющая в случае $k>1$ условию ${ }^{t} \Omega Q \Omega=0$.

ДокАЗАТЕЛЬСтво. По определению каждый многочлен $P \in \mathcal{P}_{k}^{n}(Q)$ имеет вид $P(X)=P_{0}(S X)$, где $S$ - вещественная матрица, удовлетворяющая условию $Q=2^{t} S S$, и где $P_{0} \in \mathcal{P}_{k}^{n}\left(Q_{0}\right)$ с $Q_{0}=2 \cdot 1_{m}$. Согласно следствию теории Кашивары-Вернь [11], отмеченному Э. Фрайтагом в работе [12; предложение 6.20], доказываемое предложение справедливо для $Q=Q_{0}$. Тогда оно справедливо также и для $Q={ }^{t} S Q_{0} S$, так как соотношение ${ }^{t} \Omega Q \Omega=0$ означает, что ${ }^{t}(S \Omega) Q_{0}(S \Omega)=0$. (Простое доказательство для случая $n=1$ см. в [13; гл. VI].) Предложение 3.1 доказано.

Общая линейная группа $G L_{m}(\mathbb{C})$ действует на функциях $P=P(X): \mathbb{C}_{n}^{m} \mapsto$ $\mathbb{C}$ линейными преобразованиями переменных вида

$$
U \mapsto \mid U: P(X) \mapsto(P \mid U)(X)=P(U X) \quad\left(U \in G L_{m}(\mathbb{C})\right) .
$$

Эти операторы, очевидно, сохраняют соотношения (3.4) и удовлетворяют соотношениям

$$
|U| V=\mid U V \quad\left(U, V \in G L_{m}(\mathbb{C})\right) .
$$

Лемма 3.2. Каждый из операторов $\mid U$ с $U \in G L_{m}(\mathbb{R})$ отображает пространство $\mathcal{P}_{k}^{n}(Q)$ биективно на пространство $\mathcal{P}_{k}^{n}(Q[U])$.

ДокАзАтЕльство. Если матрица $S$ удовлетворяет соотношению $2^{t} S S=Q$, то $Q[U]=2^{t}(S U)(S U)$. Таким образом, по определению,

$$
P_{Q[U]}(X)=P_{0}(S U X)=\left(P_{Q} \mid U\right)(X),
$$

где многочлен $P_{0}=P_{2 \cdot 1_{m}}(X) \in \mathcal{P}_{k}^{n}\left(Q_{0}\right)$ является гармоническим рода $n$ и веса $k$. Лемма 3.2 доказана.

Определим теперь (эрмитово) скалярное произведение функиий $P, P^{\prime}: \mathbb{R}_{n}^{m} \mapsto$ $\mathbb{C}$ относительно матриць $Q$ положительно-определенной квадратичной формы от $m$ переменных, полагая

$$
\left(P, P^{\prime}\right)=\left(P, P^{\prime}\right)_{Q}=(\operatorname{det} Q)^{\frac{n}{2}} \int_{\frac{1}{2} Q[X] \leqslant 1_{n}} P(X) \overline{P^{\prime}(X)} d X
$$


где $d X=d\left(x_{i j}\right)=\prod_{i, j} d x_{i j}$ обозначает евклидов элемент объема на $\mathbb{R}_{n}^{m}$ и неравенство $A \leqslant B$ для двух вещественных симметрических матриц одинакового порядка означает, что матрица $B-A$ является неотрицательно-полуопределенной.

Лемма 3.3. Для каждой матрицъ $U \in G L_{m}(\mathbb{R})$ и любъх функиий $P$, $P^{\prime}: \mathbb{R}_{n}^{m} \mapsto \mathbb{C}$ скалярное произведение (3.8) удовлетворяет соотношению

$$
\left(P\left|U, P^{\prime}\right| U\right)_{Q[U]}=\left(P, P^{\prime}\right)_{Q}
$$

где |U - оператор (3.6). В частности, для каждого вещественного положительного числа $\mu$ выполняется соотношение

$$
\left(P \mid \mu^{-1 / 2} U, P^{\prime}\right)_{\mu^{-1} Q[U]}=\left(P, P^{\prime} \mid \mu^{1 / 2} U^{-1}\right)_{Q} .
$$

ДокАзАтЕльство. Произведя замену переменных $X \mapsto Y=U X$, получаем

$$
\begin{aligned}
\left(P\left|U, P^{\prime}\right| U\right)_{Q[U]} & =|\operatorname{det} U|^{n}(\operatorname{det} Q)^{\frac{n}{2}} \int_{\frac{1}{2} Q[U X] \leqslant 1_{n}} P(U X) \overline{P^{\prime}(U X)} d X \\
& =(\operatorname{det} Q)^{\frac{n}{2}} \int_{\frac{1}{2} Q[Y] \leqslant 1_{n}} P(Y) \overline{P^{\prime}(Y)} d Y .
\end{aligned}
$$

Формула (3.10) следует из (3.9), если мы заменим матрицу $U$ на $\mu^{-1 / 2} U$ и функцию $P^{\prime}$ на $P^{\prime} \mid \mu^{1 / 2} U^{-1}$. Лемма 3.3 доказана.

Пусть теперь $Q$ - некоторая четная положительно-определенная матрица порядка $m$, и пусть $Q_{1}, \ldots, Q_{h}$ - некоторая система представителей из классов собственной эквивалентности (2.14), содержащихся в классе собственного подобия (2.13) матрицы $Q$, так что имеет место разложение (2.15). Гармоническим вектором рода $n$ и веса $k$ относительно системы $Q_{1}, \ldots, Q_{h}$ мы называем строку вида

$$
\mathbf{P}=\left(P_{1}, \ldots, P_{h}\right), \quad \text { где } \quad P_{i} \in \mathcal{P}_{k}^{n}\left(Q_{i}\right)
$$

Относительно обычных правил сложения строк и их умножения на комплексные числа множество

$$
\mathcal{P}_{k}^{n}\langle Q\rangle=\mathcal{P}_{k}^{n}\left(Q_{1}, \ldots, Q_{h}\right)
$$

всех гармонических векторов относительно системы $Q_{1}, \ldots, Q_{h}$, где $Q$ - некоторая матрица, содержащаяся в классе подобия матриц $Q_{i}$, может рассматриваться как линейное пространство над полем $\mathbb{C}$. Мы снабдим пространство $\mathcal{P}_{k}^{n}\langle Q\rangle$ эрмитовым скалярным произведением, определяя скалярное произведение $\left(\mathbf{P}, \mathbf{P}^{\prime}\right)$ двух векторов $\mathbf{P}=\left(P_{1}, \ldots, P_{h}\right)$ и $\mathbf{P}^{\prime}=\left(P_{1}^{\prime}, \ldots, P_{h}^{\prime}\right)$ из $\mathcal{P}_{k}^{n}\langle Q\rangle$ формулой

$$
\left(\mathbf{P}, \mathbf{P}^{\prime}\right)=\sum_{i=1}^{h}\left(P_{i}, P_{i}^{\prime}\right)_{Q_{i}}=(\operatorname{det} Q)^{n / 2} \sum_{i=1}^{h} \int_{\frac{1}{2} Q_{i}[X] \leqslant 1_{n}} P_{i}(X) \overline{P_{i}^{\prime}(X)} d X
$$

где $\left(P_{i}, P_{i}^{\prime}\right)_{Q_{i}}-$ скалярные произведения (3.8) на пространствах $\mathcal{P}_{k}^{n}\left(Q_{i}\right)$. 
Пусть теперь $\mathcal{I}_{k}^{n}\left(Q_{i}\right)$ обозначает подпространство всех многочленов из $\mathcal{P}_{k}^{n}\left(Q_{i}\right)$, которые инвариантны относительно всех операторов $\mid U$ вида (3.6) c $U \in \mathbf{E}_{i}=E^{+}\left(Q_{i}\right)$ :

$$
\mathcal{I}_{k}^{n}\left(Q_{i}\right)=\left\{P \in \mathcal{P}_{k}^{n}\left(Q_{i}\right) \mid P(U X)=P(X) \quad \text { для всех } \quad U \in \mathbf{E}_{i}\right\},
$$

И

$$
\mathcal{I}_{k}^{n}\langle Q\rangle=\mathcal{I}_{k}^{n}\left(Q_{1}, \ldots, Q_{h}\right)=\left\{\left(P_{1}, \ldots, P_{h}\right) \mid P_{i} \in \mathcal{I}_{k}^{n}\left(Q_{i}\right)\right\}
$$

- подпространство пространства $\mathcal{P}_{k}^{n}\langle Q\rangle$, состоящее из векторов, покомпонентно инвариантных относительно соответствующих групп единиц. Кольцо классов автоморфов $\mathcal{H}\langle Q\rangle=\mathcal{H}\left(Q_{1}, \ldots, Q_{h}\right)$ естественно действует на пространстве $\boldsymbol{I}_{k}^{n}$ линейными операторами: для $\boldsymbol{\tau}=\left(\tau_{i j}\right) \in \mathcal{H}\langle Q\rangle$ мы определим оператор Гекке $\mid \boldsymbol{\tau}$ на $\mathcal{I}_{k}^{n}$, полагая

$$
|\boldsymbol{\tau}=|\left(\tau_{i j}\right) \mathbf{P}=\left(P_{i}\right) \mapsto \mathbf{P} \mid \boldsymbol{\tau}=\left(\sum_{i=1}^{h} P_{i}\left|\tau_{i 1}, \ldots, \sum_{i=1}^{h} P_{i}\right| \tau_{i h}\right),
$$

где для $\tau_{i j}=\sum_{\alpha} a_{\alpha}\left(\mathbf{E}_{i} D_{\alpha}\right) \in \mathcal{H}\langle Q\rangle_{i j}$ с $D_{\alpha} \in \mathbf{A}_{i j}$ (см. (2.17)) и $P_{i} \in \mathcal{I}_{k}^{n}\left(Q_{i}\right)$ использовано обозначение

$$
P_{i}\left|\tau_{i j}=\sum_{\alpha} a_{\alpha} P_{i}\right| D_{\alpha} \in \mathcal{P}_{k}^{n}\left(Q_{j}\right)
$$

и где операторы $\mid D_{\alpha}$ определяются формулой (3.6). Так как $P_{i} \in \mathcal{I}_{k}^{n}\left(Q_{i}\right)$, то каждый из многочленов (3.16) не зависит от выбора представителей $D_{\alpha}$ в классе $\mathbf{E}_{i} D_{\alpha}$. Так как $\tau_{i j} U=\tau_{i j}$ для всех $U \in \mathbf{E}_{j}$, мы заключаем, что каждый из многочленов (3.16), фактически, принадлежит пространству $\mathcal{I}_{k}^{n}\left(Q_{j}\right)$. Поэтому каждый из операторов $\mid \boldsymbol{\tau}$ отображает подпространство $\mathcal{I}_{k}^{n}\langle Q\rangle$ в себя. Операторы $\mid \boldsymbol{\tau}$, очевидно, линейны и, как легко следует из определения умножения в кольце $\mathcal{H}\langle Q\rangle$, оператор, соответствующий произведению двух элементов кольца $\mathcal{H}\langle Q\rangle$, равен произведению операторов, отвечающих сомножителям:

$$
\left|\boldsymbol{\tau} \boldsymbol{\tau}^{\prime}=\right| \boldsymbol{\tau} \mid \boldsymbol{\tau}^{\prime} \quad\left(\boldsymbol{\tau}, \boldsymbol{\tau}^{\prime} \in \mathcal{H}\langle Q\rangle\right) .
$$

Таким образом, мы получаем линейное представление кольца $\mathcal{H}\langle Q\rangle$ на пространстве $\mathcal{I}_{k}^{n}\langle Q\rangle$.

Теорема 3.4. Пусть $\mathcal{H}_{r}\langle Q\rangle=D\left(\mathbf{E}_{1}, \ldots, \mathbf{E}_{h} ; \mathbf{S}_{11}, \mathbf{S}_{12}, \ldots, \mathbf{S}_{h h}\right)-$ кольио классов подобия некоторой четной положительно-определенной матрицы $Q$.

Предположим, что порядки групп единии $\mathbf{E}_{1}, \ldots, \mathbf{E}_{h}$ равны друг другу. Тогда для каждого элемента $\boldsymbol{\tau}$ кольиа $\mathcal{H}_{r}\langle Q\rangle$ операторы Гекке $|\boldsymbol{\tau} u| \boldsymbol{\tau}^{*}$ на пространстве $\boldsymbol{I}_{k}^{n}\langle Q\rangle$, где $\boldsymbol{\tau}^{*}$ - элемент $(2.22)$, сопряљены относительно скалярного произведения (3.13):

$$
\left(\mathbf{P} \mid \boldsymbol{\tau}, \mathbf{P}^{\prime}\right)=\left(\mathbf{P}, \mathbf{P}^{\prime} \mid \boldsymbol{\tau}^{*}\right) \quad\left(\mathbf{P}, \mathbf{P}^{\prime} \in \mathcal{I}_{k}^{n}\langle Q\rangle, \quad \boldsymbol{\tau} \in \mathcal{H}_{r}\langle Q\rangle\right) .
$$

ДокАЗАТЕЛЬСтво. Достаточно доказать соотношения (3.17) для элементов $\boldsymbol{\tau}=\left(\tau\left(D_{i j}\right)\right)$ вида $(2.19)$ с $D_{i j} \in \mathbf{S}_{i j}$. В этом случае, используя $(2.22)$, получаем

$$
\boldsymbol{\tau}^{*}=\left(\tau\left(D_{i j}\right)\right)^{*}={ }^{t}\left(\tau\left(D_{i j}^{*}\right)\right)={ }^{t}\left(\tau\left(\mu\left(D_{i j}\right) D_{i j}^{-1}\right)\right) .
$$


По лемме 2.1 каждый двойной класс $\mathbf{E}_{i} D_{i j} \mathbf{E}_{j}$ содержит общую систему представителей $\left\{D_{i j}^{\alpha}\right\}$ из левых классов по модулю $\mathbf{E}_{i}$ и правых классов по модулю $\mathbf{E}_{j}$. Тогда, используя лемму 2.2 , мы легко заключаем, что система $\left\{\mu\left(D_{i j}\right)\left(D_{i j}^{\alpha}\right)^{-1}\right\}$ является общей системой представителей из левых классов по модулю $\mathbf{E}_{j}$ и правых классов по модулю $\mathbf{E}_{i}$, содержащихся в двойном классе $\mathbf{E}_{j} \mu\left(D_{i j}\right) D_{i j}^{-1} \mathbf{E}_{i}$. В частности, для этой системы представителей имеют место разложения

$$
\tau\left(D_{i j}\right)=\sum_{\alpha}\left(\mathbf{E}_{i} D_{i j}^{\alpha}\right) \quad \text { и } \quad \tau\left(\mu\left(D_{i j}\right) D_{i j}^{-1}\right)=\sum_{\alpha}\left(\mathbf{E}_{j} \mu\left(D_{i j}\right)\left(D_{i j}^{\alpha}\right)^{-1}\right) .
$$

Если $\mathbf{P}=\left(P_{1}, \ldots, P_{h}\right)$, то на основании формул $(3.15),(3.16)$ и (3.18) мы получаем

$$
\mathbf{P} \mid \boldsymbol{\tau}=\left(\sum_{i=1}^{h} \sum_{\alpha} P_{i}\left|D_{i 1}^{\alpha}, \ldots, \sum_{i=1}^{h} \sum_{\alpha} P_{i}\right| D_{i h}^{\alpha}\right),
$$

где $\mid D_{i j}^{\alpha}$ - операторы (3.6). Отсюда, согласно (3.13), следует соотношение

$$
\left(\mathbf{P} \mid \tau, \mathbf{P}^{\prime}\right)=\sum_{j=1}^{h} \sum_{i=1}^{h} \sum_{\alpha}\left(P_{i} \mid D_{i j}^{\alpha}, P_{j}^{\prime}\right)_{Q_{j}} .
$$

Используя снова формулы (3.15), (3.16) и (3.18), мы можем написать

$$
\mathbf{P}^{\prime} \mid \boldsymbol{\tau}^{*}=\left(\sum_{i=1}^{h} \sum_{\alpha} P_{i}^{\prime}\left|\mu\left(D_{1 i}\right)\left(D_{1 i}^{\alpha}\right)^{-1}, \ldots, \sum_{i=1}^{h} \sum_{\alpha} P_{i}^{\prime}\right| \mu\left(D_{h i}\right)\left(D_{h i}^{\alpha}\right)^{-1}\right),
$$

откуда

$$
\left(\mathbf{P}, \mathbf{P}^{\prime} \mid \boldsymbol{\tau}^{*}\right)=\sum_{j=1}^{h} \sum_{i=1}^{h} \sum_{\alpha}\left(P_{j}, P_{i}^{\prime} \mid \mu\left(D_{j i}\right)\left(D_{j i}^{\alpha}\right)^{-1}\right)_{Q_{j}} .
$$

По (3.4) и (3.10) мы получаем

$$
\begin{gathered}
\left(P_{j}, P_{i}^{\prime} \mid \mu\left(D_{j i}\right)\left(D_{j i}^{\alpha}\right)^{-1}\right)_{Q_{j}}=\mu\left(D_{j i}\right)^{n k / 2}\left(P_{j}, P_{i}^{\prime} \mid \mu\left(D_{j i}\right)^{1 / 2}\left(D_{j i}^{\alpha}\right)^{-1}\right)_{Q_{j}} \\
=\mu\left(D_{j i}\right)^{n k / 2}\left(P_{j} \mid \mu\left(D_{j i}\right)^{-1 / 2} D_{j i}^{\alpha}, P_{i}^{\prime}\right)_{\mu\left(D_{j i}\right)^{-1} Q_{j}\left[D_{j i}\right]}=\left(P_{j} \mid D_{j i}^{\alpha}, P_{i}^{\prime}\right)_{Q_{i}} .
\end{gathered}
$$

Отсюда следует, что

$$
\left(\mathbf{P}, \mathbf{P}^{\prime} \mid \boldsymbol{\tau}^{*}\right)=\sum_{i, j=1}^{h} \sum_{\alpha}\left(P_{j} \mid D_{j i}^{\alpha}, P_{i}^{\prime}\right)=\left(\mathbf{P} \mid \boldsymbol{\tau}, \mathbf{P}^{\prime}\right) .
$$

Теорема 3.4 доказана.

ПРЕДЛОЖЕНИЕ 3.5. В обозначениях и предположениях теоремы 3.4 операторы Гекке $\mid \boldsymbol{\tau}$ на каждом инвариантном подпространстве пространства $\mathcal{I}_{k}^{n}\langle Q\rangle$, отвечающие любой системе коммутирующих друг с другом элементов $\boldsymbol{\tau} \in \mathcal{H}_{r}\langle Q\rangle$, подчиненных условию $\boldsymbol{\tau}^{*}=\boldsymbol{\tau}$, могут быть одновременно диагонализированы. В частности, в случалх $m=2$ или $m=4$ операторы Гекке, отвечаюшие элементам $\boldsymbol{\tau}\left(p^{\delta}\right)$ с простым $p$, не делящем $\operatorname{det} Q, u \delta=0,1,2, \ldots$, могут быть одновременно диагонализированы на каждом инвариантном подпространстве. 
ДоказАТЕЛЬство. По теореме 3.4 операторы Гекке $\mid \boldsymbol{\tau}$ для элементов $\boldsymbol{\tau}$, удовлетворяющих условию $\tau^{*}=\boldsymbol{\tau}$, самосопряжены относительно эрмитова скалярного произведения (3.13). По известной теореме линейной алгебры любое семейство коммутирующих друг с другом самосопряженных линейных операторов на конечномерном гильбертовом пространстве может быть одновременно диагонализировано. Последнее утверждение следует из первого и предположения 2.8. Предложение 3.5 доказано.

Можно предположить, что линейные комбинации с целочисленными коэффициентами элементов вида (2.28), содержащихся в кольце $\boldsymbol{H}_{r}\langle Q\rangle$, образуют подкольцо. Если это верно, то из лемм 2.4 и 2.5 следует, что это подкольцо коммутативно. В настоящее время это предположение доказано только для квадратичных форм от $m=2$ переменных (см. [7; теорема 2.4]).

\section{§ 4. Действие операторов Гекке на гармонические тета-суммы}

Пусть заданы четная положительно-определенная матрица $Q$ порядка $m$ и система представителей $Q_{1}, \ldots, Q_{h}$ из классов эквивалентности $(2.14)$, содержащихся в классе подобия (2.13) матрицы $Q$, и пусть $\mathbf{P}=\left(P_{1}, \ldots, P_{h}\right)$ - некоторый гармонический вектор (3.11) рода $n$ и веса $k$ относительно системы $Q_{1}, \ldots, Q_{h}$. Мы определим гармоническую тета-сумму рода $n$ класса подобия $\langle Q\rangle=\langle Q\rangle^{+}$, отвечающую вектору $\mathbb{P}$, формулой

$$
\Theta(Z ; \mathbf{P},\langle Q\rangle)=\theta\left(Z ; P_{1}, Q_{1}\right)+\cdots+\theta\left(Z ; P_{h}, Q_{h}\right),
$$

где переменная $Z$ принадлежит верхней полуплоскости рода $n$,

$$
\mathbb{H}_{n}=\left\{Z=X+\left.i Y \in \mathbb{C}_{n}^{n}\right|^{t} Z=Z, Y>0\right\},
$$

и где функция

$$
\theta\left(Z ; P_{i}, Q_{i}\right)=\sum_{N \in \mathbb{Z}_{n}^{m}} P_{i}(N) e^{\pi \sqrt{-1} \operatorname{Trace}\left(Q_{i}[N] Z\right)}
$$

является тета-рядом рода $n$ квадратичной формы с матрицей $Q_{i}$, соответствующим гармонической форме $P_{i}$. Каждый из этих тета-рядов, очевидно, сходится абсолютно и равномерно на компактных подмножествах верхней полуплоскости $\mathbb{H}_{n}$ и, таким образом, определяет голоморфную функцию от $n(n+1) / 2$ комплексных переменных. Разложение Фурье такой функции имеет вид

$$
\theta\left(Z ; P_{i}, Q_{i}\right)=\sum_{A \in \mathbb{E}_{n}, A \geqslant 0} r\left(A ; P_{i}, Q_{i}\right) e^{\pi \sqrt{-1} \operatorname{Trace}(A Z)}
$$

с постоянными коэффициентами Фурье вида

$$
r\left(A ; P_{i}, Q_{i}\right)=\sum_{N \in \mathbb{Z}_{n}^{m}, Q_{i}[N]=A} P_{i}(N) .
$$

Заменяя $N$ на $U N$ с $U \in \Lambda^{m}=G L_{m}(\mathbb{Z})$, получаем тождество

$$
\theta\left(Z ; P_{i} \mid U,{ }^{t} U Q_{i} U\right)=\theta\left(Z ; P_{i}, Q_{i}\right),
$$


где многочлен $P_{i} \mid U$ определен формулой (3.6); в частности,

$$
\theta\left(Z ; P_{i} \mid U, Q_{i}\right)=\theta\left(Z ; P_{i}, Q_{i}\right) \quad \text { для всех } U \in \mathbf{E}_{i} \text {. }
$$

Заменяя, если это необходимо, каждый из многочленов $P_{i}$ его усреднением

$$
\left[\#\left(\mathbf{E}_{i}\right)\right]^{-1} \sum_{U \in \mathbf{E}_{i}} P_{i} \mid U
$$

по группе единиц $\mathbf{E}_{i}$, что не меняет тета-ряда, мы можем, не умаляя общности, предполагать, что $P_{i} \in \mathcal{I}_{k}^{n}\left(Q_{i}\right)$ для $i=1, \ldots, h$, т.е. что $\mathbf{P} \in \mathcal{I}_{k}^{n}\langle Q\rangle$.

Согласно работе [14] (см. также [15]), если $m$ четно, то каждый из тетарядов (4.2) принадлежит пространству $\mathfrak{M}_{m / 2+k}^{n}\left(q, \chi_{Q}\right)$ модулярных форм веса $m / 2+k$ для группы

$$
\Gamma_{0}^{n}(q)=\left\{M=\left(\begin{array}{ll}
A & B \\
C & D
\end{array}\right) \in \operatorname{Sp}_{n}(\mathbb{Z}) \mid C \equiv 0(\bmod q)\right\},
$$

где $q$ - ступень матрицы $Q$, с характером (Дирихле) $\chi_{Q}$ по модулю $q$, удовлетворяющим условиям $\chi_{Q}(-1)=(-1)^{m / 2}$ и

$$
\chi_{Q}(p)=\left(\frac{(-1)^{m / 2} \operatorname{det} Q}{p}\right) \quad \text { (символ Лежандра), }
$$

если $p$ - нечетное простое число, не делящее ступень $q$. В частности, функция $F=F(Z)=\theta\left(Z ; P_{j}, Q_{j}\right)$ удовлетворяет функциональному уравнению

$$
\operatorname{det}(C Z+D)^{-(m / 2+k)} F\left((A Z+B)(C Z+D)^{-1}\right)=\chi_{Q}(\operatorname{det} D) F(Z)
$$

для каждой матрицы $\left(\begin{array}{ll}A & B \\ C & D\end{array}\right) \in \Gamma_{0}^{n}(q)$. Поэтому тета-сумма (4.1) также является модулярной формой,

$$
\Theta(Z ; \mathbf{P},\langle Q\rangle) \in \mathfrak{M}_{m / 2+k}^{n}\left(q, \chi_{Q}\right) .
$$

Следуя общей схеме теории операторов Гекке на пространстве зигелевых модулярных форм (см., например, [9; гл. 4] или [6; § 2]), мы напомним теперь основные определения и простейшие свойства (регулярных) операторов Гекке на пространствах $\mathfrak{M}_{w}^{n}(q, \chi)$ модулярных форм целого веса $w$ и характера $\chi$ для группы $\Gamma_{0}^{n}(q)$. Обозначим через

$$
\mathcal{H}_{0}^{n}(q)=\mathcal{H}\left(\Gamma_{0}^{n}(q), \Sigma_{0}^{n}(q)\right)
$$

кольцо Гекке-Шимуры (над $\mathbb{C}$ ) полугруппы

$$
\begin{aligned}
\Sigma_{0}^{n}(q)= & \left\{M=\left(\begin{array}{ll}
A & B \\
C & D
\end{array}\right) \in \mathbb{Z}_{2 n}^{2 n} \mid{ }^{t} M J_{n} M=\mu(M) J_{n}, \mu(M)>0,\right. \\
& \operatorname{gcd}(\operatorname{det} M, q)=1, C \equiv 0(\bmod q)\} \quad\left(J_{n}=\left(\begin{array}{cc}
0 & 1_{n} \\
-1_{n} & 0
\end{array}\right)\right)
\end{aligned}
$$


относительно группы $\Gamma_{0}^{n}(q)$. Кольцо $\mathcal{H}_{0}^{n}(q)$ порождено над $\mathbb{C}$ коммутирующими друг с другом алгебраически независимыми элементами

$$
\left\{\begin{array}{l}
T^{n}(p)=(\operatorname{diag}(\underbrace{1, \ldots, 1}_{n}, \underbrace{p, \ldots, p}_{n}))_{\Gamma_{0}^{n}(q)}, \\
T_{j}^{n}\left(p^{2}\right)=\left(\operatorname{diag}(\underbrace{1, \ldots, 1}_{n-j}, \underbrace{p, \ldots, p}_{j}, \underbrace{p^{2}, \ldots, p^{2}}_{n-j}, \underbrace{p, \ldots, p}_{j})_{\Gamma_{0}^{n}(q)} \quad(1 \leqslant j \leqslant n),\right.
\end{array}\right.
$$

где $p$ пробегает все простые числа, не делящие ступень $q$, и где

$$
(M)_{\Gamma}=\tau(M)=\sum_{M^{\prime} \in \Gamma \backslash \Gamma M \Gamma}\left(\Gamma M^{\prime}\right) \quad\left(\Gamma=\Gamma_{0}^{n}(q), M \in \Sigma_{0}^{n}(q)\right)
$$

обозначает двойной класс (2.5) матриць $M$ по модулю $\Gamma_{0}^{n}(q)$ (см. [9; теорема 3.3.23]).

Оператор Гекке $|T=|_{w, \chi} T$ на пространстве $\mathfrak{M}_{w}^{n}(q, \chi)$ для

$$
T=\sum_{i} c_{i}\left(\Gamma_{0}^{n}(q) M_{i}\right) \in \mathcal{H}_{0}^{n}(q)
$$

можно определить формулой

$$
F\left|T=\sum_{i} c_{i} F\right|_{w, \chi} M_{i} \quad\left(F \in \mathfrak{M}_{w}^{n}(q, \chi)\right),
$$

где

$$
\begin{gathered}
\left.F\right|_{w, \chi}\left(\begin{array}{ll}
A & B \\
C & D
\end{array}\right)=\chi(\operatorname{det} A)[\operatorname{det}(C Z+D)]^{-w} F\left((A Z+B)(C Z+D)^{-1}\right) \\
\left(\left(\begin{array}{ll}
A & B \\
C & D
\end{array}\right) \in \Sigma_{0}^{n}(q)\right)
\end{gathered}
$$

обозначают операторы Петерссона. Операторы Гекке не зависят от выбора представителей $M_{i} \in \Gamma_{0}^{n}(q) M_{i}$ и отображают пространство $\mathfrak{M}_{w}^{n}(q, \chi)$ в себя.

Довольно часто операторы Гекке отображают тета-ряды в линейные комбинации аналогичных тета-рядов. Легкое видоизменение частного случая основного результата работы [6; теорема 4.1] может быть сформулировано следующим образом. Пусть $Q$ - четная положительно-определенная матрица четного порядка $m$ и $q$ - ее ступень. Предположим, что двойной класс $\tau(M) \in \mathcal{H}_{0}^{n}(q)$ вида $(4.6)$ с $\mu(M)=\mu$, где $n \leqslant m$, содержится в образе кольца $\mathcal{H}_{0}^{m}(q)$ при отображении Жарковской

$$
\Psi^{m, n}=\Psi_{Q}^{m, n}=\Psi_{m / 2, \chi_{Q}}: \mathcal{H}_{0}^{m}(q) \mapsto \mathcal{H}_{0}^{n}(q)
$$

(см. [9; §4.2.4] и $[6 ; \S 3])$. Тогда образ тета-ряда $\theta\left(Z, P_{i}, Q_{i}\right)$ рода $n$ каждой положительно-определенной матрицы $Q_{i}$, подобной матрице $Q$, с гармонической формой $P_{i} \in \mathcal{I}_{k}^{n}\left(Q_{i}\right)$ под действием оператора Гекке, отвечающего двойному классу $\tau(M) \in \mathcal{H}_{0}^{n}(q)$, может быть записан в виде

$$
\theta\left(Z ; P_{i}, Q_{i}\right) \mid \tau(M)=\sum_{D \in S\left(Q_{i}, \mu\right) / \Lambda_{+}} I\left(D, Q_{i}, \Psi^{n, m}(\tau(M))\right) \theta\left(Z ; P_{i} \mid \mu^{-1} D, \mu^{-1} Q_{i}[D]\right),
$$


где

$$
S\left(Q_{i}, \mu\right)=\left\{D \in \mathbb{Z}_{m}^{m} \mid \operatorname{det} D=\mu^{m / 2}, \mu^{-1} Q_{i}[D] \in \mathbb{E}_{m}\right\},
$$

$\Lambda_{+}=S L_{m}(\mathbb{Z}), \Psi^{n, m}(M) \in \mathcal{H}_{0}^{m}(q)$ обозначает прообраз двойного класса $\tau(M)$ при отображении $\Psi^{m, n}$ и где операторы $P \mapsto P \mid U$ определяются формулой (3.6); при этом $I\left(D, Q^{\prime}, T\right)$ - некоторые постоянные (т.е. не зависящие от $Z$ и $P_{i}$ ) коэффициенты, удовлетворяющие соотношениям

$$
I\left(U D V, Q^{\prime}, T\right)=I\left(D, Q^{\prime}[U], T\right)
$$

для всех $U, V \in \Lambda=G L_{m}(\mathbb{Z}), Q^{\prime} \in\langle Q\rangle$ и $T \in \mathcal{H}_{0}^{m}(q)$.

Каждая из матриц $\mu^{-1} Q_{i}[D]$ с $D \in S\left(Q_{i}, \mu\right)$ собственно подобна матрице $Q_{i} \simeq^{+} Q$ и поэтому собственно эквивалентна одному из представителей из классов эквивалентности $\left\{Q_{1}\right\}^{+}, \ldots,\left\{Q_{h}\right\}^{+}$, содержащихся в классе подобия $\langle Q\rangle=\langle Q\rangle^{+}$, скажем, матрице $Q_{j}$, т.е. $\mu^{-1}[D]=Q_{j}[U]$ с $U \in \Lambda_{+}$. Отсюда следует, что формулу для действия оператора $\mid \tau(M)$ можно переписать в виде

$$
\theta\left(Z ; P_{i}, Q_{i}\right) \mid \tau(M)=\mu^{-n k} \sum_{j=1}^{h} \sum_{D \in R\left(Q_{i}, \mu Q_{j}\right) / \mathbf{E}_{j}} I\left(D, Q_{i}, \Psi^{n, m}(\tau(M))\right) \theta\left(Z ; P_{i} \mid D, Q_{j}\right),
$$

где мы также использовали соотношения (3.4).

Следующие формулы суммируют все известные в настоящее время результаты вычислений коэффициентов $I\left(D, Q^{\prime}, T\right)$ с $Q^{\prime} \in\langle Q\rangle$ : для каждого простого числа $p$, не делящего ступень $q$ матрицы $Q$, имеют место формулы

$$
I\left(D, Q^{\prime}, T^{m}(p)\right)= \begin{cases}p^{m / 2} \prod_{j=1}^{m / 2}\left(1+\chi_{Q}(p) p^{-j}\right), & \text { если } D \in \Lambda D_{m / 2}^{m}(p) \Lambda, \\ 0 & \text { в остальных случаях, }\end{cases}
$$

где $\Lambda=G L_{m}(\mathbb{Z}), D_{m / 2}^{m}(p)=\operatorname{diag}(\underbrace{1, \ldots, 1}_{m / 2}, \underbrace{p, \ldots, p}_{m / 2})$;

$$
I\left(D, Q^{\prime}, T_{m-1}^{m}\left(p^{2}\right)\right)= \begin{cases}\chi_{Q}(p) p^{\left(2+m-m^{2}\right) / 2}, & \text { если } D \in \Lambda D_{m-2,1}^{m}(p) \Lambda, \\ \alpha_{m}(p), & \text { если } D \in \Lambda\left(p 1_{m}\right), \\ 0 & \text { в остальных случаях, }\end{cases}
$$

где $D_{m-2,1}^{m}(p)=\operatorname{diag}(1, \underbrace{p, \ldots, p}_{m-2}, p^{2})$ и

$$
\begin{gathered}
\alpha_{m}(p)=\chi_{Q}(p) p^{\left(2+m-m^{2}\right) / 2} \frac{\left(p^{m}-1\right)}{p-1}+p^{-m^{2} / 2}\left(\chi_{Q}(p) p^{m / 2}-1\right) ; \\
I\left(D, Q^{\prime},\langle p\rangle_{m}\right)= \begin{cases}p^{-m^{2} / 2}, & \text { если } D \in \Lambda\left(p 1_{m}\right), \\
0 & \text { в противном случае },\end{cases}
\end{gathered}
$$

где для краткости мы пишем

$$
T_{n}^{n}\left(p^{2}\right)=\left(p \cdot 1_{2 n}\right)_{\Gamma_{0}^{n}(q)}=\langle p\rangle_{n} .
$$

(В работе [4; формула (2.19) и лемма 5.1] суммы $\gamma(Q, D, T)$, аналогичные коэффициентам $I(D, Q, T)$, были определены и вычислены для элементов $T=$ 
$T^{m}(p)$. В работе $[10 ; \S 2]$ суммы $\gamma(Q, D, T)$ были, фактически, вычислены для элементов $T=\langle p\rangle_{m}=T_{m}^{m}\left(p^{2}\right)=\left(p 1_{2 m}\right)_{\Gamma_{0}^{m}(q)}$ и $T=T_{m-1}^{m}\left(p^{2}\right)$. См. также [9; лемма 3.3.32] по поводу использованного в работе [10] представления элемента $T_{m-1}^{m}\left(p^{2}\right)$. Непосредственно из определения этих сумм следуют формулы $I(D, Q, \tau(M))=\chi_{Q}(\mu)^{m} \mu^{m / 2} \gamma\left(Q, \mu D^{-1}, \tau(M)\right)=\mu^{m / 2} \gamma\left(Q, \mu D^{-1}, \tau(M)\right)$, где $\mu=\mu(M)$.)

Из формул (4.10)-(4.12) следует, в частности, что для элементов

$$
T=T^{m}(p), \quad\langle p\rangle_{m}=T_{m}^{m}\left(p^{2}\right) \quad \text { и } \quad T_{m-1}^{m}\left(p^{2}\right)
$$

с простыми $p$, не делящими ступень матрицы $Q$, коэффициенты $I\left(D, Q^{\prime}, T\right)$ как функции от $Q^{\prime}$ зависят только от класса подобия матрицы $Q^{\prime}$, а как функции от $D$ зависят только от двойного класса $\Lambda D \Lambda$. Поэтому, если $\Psi^{n, m}(\tau(M))-$ некоторая линейная комбинация элементов (4.13), то при тех же предположениях формулу (4.9) можно переписать в виде

$$
\begin{aligned}
\theta\left(Z ; P_{i}, Q_{i}\right) \mid \tau(M)=\mu^{-n k} & \sum_{\substack{d_{1}|\cdots| d_{m} ; \\
d_{i} d_{m-i+1=\mu}}} I\left(\operatorname{diag}\left(d_{1}, \ldots, d_{m}\right), Q, \Psi^{n, m}(\tau(M))\right) \\
& \times \sum_{j=1}^{h} \theta\left(Z ; \sum_{D \in \mathbf{S}_{i j} \cap \Lambda_{+} \operatorname{diag}\left(d_{1}, \ldots, d_{m}\right) \Lambda_{+} / \mathbf{E}_{j}} P_{i} \mid D, Q_{j}\right) .
\end{aligned}
$$

Предположим теперь, что порядки групп $\mathbf{E}_{1}, \ldots, \mathbf{E}_{h}$ равны друг другу, тогда по лемме 2.1 кольцо $\mathcal{H}_{r}\langle Q\rangle$ является ручным, и мы можем взять в качестве системы представителей из правых классов по модулю $\mathbf{E}_{j}$, содержащихся в любом двойном классе $\mathbf{E}_{i} D^{\prime} \mathbf{E}_{j} \subset R^{+}\left(Q_{i}, \mu Q_{j}\right)$, подходящую систему представителей из левых классов $\mathbf{E}_{i} \backslash \mathbf{E}_{i} D^{\prime} \mathbf{E}_{j}$. Это позволяет переписать последнюю формулу в виде

$$
\begin{aligned}
\theta\left(Z ; P_{i}, Q_{i}\right) \mid \tau(M)= & \mu^{-n k} \sum_{\substack{d_{1}|\cdots| d_{m} ; \\
d_{i} d_{m-i+1}=\mu}} I\left(\operatorname{diag}\left(d_{1}, \ldots, d_{m}\right), Q, \Psi^{n, m}(\tau(M))\right) \\
& \times \sum_{j=1}^{h} \theta\left(Z ; \sum_{\substack{D \in \mathbf{E}_{i} \backslash \mathbf{S}_{i j} \cap \Lambda_{+} \operatorname{diag}\left(d_{1}, \ldots, d_{m}\right) \Lambda_{+} \\
=}} P_{i} \mid D, Q_{j}\right) \\
& \times \sum_{j=1} I\left(\operatorname{diag}\left(d_{1}, \ldots, d_{m}\right), Q, \Psi^{n, m}(\tau(M))\right) \\
& \sum_{\substack{d_{1}|\cdots| d_{m} ; \\
d_{i} d_{m-i+1}=\mu}}^{h} \theta\left(Z ; P_{i} \mid \tau_{i j}\left[d_{1}, \ldots, d_{m}\right], Q_{j}\right)
\end{aligned}
$$

(см. (2.28) и (3.16)). Возвращаясь к тета-суммам, при тех же предположениях мы можем представить образ тета-суммы (4.1) под действием оператора $\mid \tau(M)$ 
в виде

$$
\begin{aligned}
& \Theta(Z ; \mathbf{P}, Q)\left|\tau(M)=\theta\left(Z ; P_{1}, Q_{1}\right)\right| \tau(M)+\cdots+\theta\left(Z ; P_{h}, Q_{h}\right) \mid \tau(M) \\
& =\mu^{-n k} \sum_{\begin{array}{c}
d_{1}|\cdots| d_{m} ; \\
d_{i} d_{m-i+1}=\mu
\end{array}} I\left(\operatorname{diag}\left(d_{1}, \ldots, d_{m}\right), Q, \Psi^{n, m}(M)\right) \\
& \quad \times \sum_{j=1}^{h} \theta\left(Z ; \sum_{i=1}^{h} P_{i} \mid \tau_{i j}\left[d_{1}, \ldots, d_{m}\right], Q_{j}\right) \\
& =\mu^{-n k} \sum_{\substack{d_{1}|\cdots| d_{m} ; \\
d_{i} d_{m-i+1}=\mu}} I\left(\operatorname{diag}\left(d_{1}, \ldots, d_{m}\right), Q, \Psi^{n, m}(\tau(M))\right) \Theta\left(Z ; \mathbf{P} \mid \boldsymbol{\tau}\left[d_{1}, \ldots, d_{h}\right], Q\right)
\end{aligned}
$$

(см. (2.28) и (3.15)).

Формулы (4.10)-(4.12) определяют, в частности, суммы $I(D, Q, T)$ для всех образующих колец $\mathcal{H}_{0}^{1}(q)$ и $\mathcal{H}_{0}^{2}(q)$, при условии, что мы можем явно выразить прообразы $\Psi^{n, m}(\tau(M))$ образующих (4.5) для $n=1,2$ через элементы (4.13). С этой целью мы сначала рассмотрим действие отображения Жарковской на соответствующие элементы.

Лемма 4.1. Следующие формуль имеют место для действия отображения Жарковской $\Psi=\Psi_{w, \chi}^{n, n-1}: \mathcal{H}_{0}^{n}(q) \mapsto \mathcal{H}_{0}^{n-1}(q)$ на некоторые из элементов вида (4.5) для $n>1$ и каждого простого числа $p$, не делящего $q$ :

$$
\begin{aligned}
\Psi^{n, n-1}\left(T^{n}(p)\right) & =\left(1+\bar{\chi}(p) p^{n-w}\right) T^{n-1}(p) ; \\
\Psi^{n, n-1}\left(\langle p\rangle_{n}\right) & =\bar{\chi}(p) p^{-w}\langle p\rangle_{n-1} ; \\
\Psi^{n, n-1}\left(T_{n-1}^{n}\left(p^{2}\right)\right) & =\bar{\chi}(p) p^{1-w} T_{n-2}^{n-1}\left(p^{2}\right)+b_{n}(p)\langle p\rangle_{n-1},
\end{aligned}
$$

где $b_{n}(p)=b_{n, w, \chi}(p)=\bar{\chi}\left(p^{2}\right) p^{2 n-2 w}+\bar{\chi}(p)(p-1) p^{-w}+1$.

ДокАЗАтЕльство. Действие отображения Жарковской, отвечающего действию операторов Гекке на пространствах $\mathfrak{M}_{w}^{n}(q, \chi)$, было вычислено в книге $[9 ; \S 4.2 .4]$. Однако, применяя результаты этих вычислений, необходимо учитывать, что операторы Гекке, определенные в [9; (2.4.11) и (2.4.12)] имеют нормализацию, отличную от той, которую мы используем в настоящей работе, и отличаются от операторов, определенных в работе [6; формулы $(1.10)$ с $l=0$, $(2.13),(2.14)$ и $(2.20)$ с $Q=H$ и $P=0]$ для однородных элементов мультипликатора $\mu$, множителем $\chi\left(\mu^{n}\right) \mu^{n w-n(n+1) / 2}$.

Формула (4.15) следует из результатов книги [9; предложения 4.2.17 и 4.2.18 и формула (4.2.80)] для операторов $\left.\right|_{w} \chi\left(p^{n}\right) p^{n w-n(n+1) / 2} T^{n}(p)$ (учтите отмеченное выше различие нормализаций). Формула (4.16) следует по аналогичным причинам из [9; лемма 3.3.34]. Что касается формулы (4.17), то здесь ситуация несколько сложнее. Прежде всего, используя [9; разложение (3.5.69) теорема 3.5.23, формулы (3.5.34), (3.4.15), (3.5.33) и (3.3.61)], мы получаем соотношение

$$
T_{n-1}^{n}\left(p^{2}\right)=-p^{n}\langle p\rangle_{n} \mathbf{r}_{1}^{n}(p)+\left(p^{n}-1\right)\langle p\rangle_{n},
$$


где $\mathbf{r}_{1}^{n}(p)$ обозначает первый коэффициент $p$-многочлена Ранкина $R_{p}^{n}(v)$, определенного в [9; формулы (3.5.15) и (3.5.16)]. Так как $\mu\left(\mathbf{r}_{1}^{n}(p)\right)=1$, то из результатов [9; теорема 4.2 .18 и соотношение (4.2.82)] следует соотношение

$$
\Psi^{n, n-1}\left(\mathbf{r}_{1}^{n}(p)\right)=\mathbf{r}_{1}^{n-1}(p)-\bar{\chi}(p) p^{n-w}-\chi(p) p^{w-n}
$$

Поскольку отображение Жарковской является кольцевым гомоморфизмом, формула (4.17) следует легким вычислением из (4.18), (4.16), (4.19). Лемма 4.1 доказана.

Последовательно применяя формулу (4.15) к элементам $T^{m}(p), \ldots, T^{n}(p)$ с $w=m / 2, \chi=\chi_{Q}$ и $n<m$, мы получаем соотношение

$$
\Psi^{m, n}\left(T^{m}(p)\right)=\gamma_{n}^{m}(p) T^{n}(p), \quad \text { где } \quad \gamma_{n}^{m}(p)=\left\{\prod_{i=0}^{m-n-1}\left(1+\chi_{Q}(p) p^{m / 2-i}\right)\right\} .
$$

Множитель $\gamma_{n}^{m}(p)$ равен 0 в том и только том случае, если $m / 2 \leqslant m-n-1$, т.е. $n \leqslant m / 2-1$, и $\chi_{Q}(p)=-1$. Отсюда следует соотношение

$$
\Psi^{n, m}\left(T^{n}(p)\right)=\gamma_{n}^{m}(p)^{-1} T^{m}(p),
$$

кроме случая, когда $n \leqslant m / 2-1$ и $\chi_{Q}(p)=-1$. Аналогично, используя (4.16), получаем $\Psi^{m, n}\left(\langle p\rangle_{m}\right)=\left(\chi_{Q}(p) p^{-m / 2}\right)^{m-n}\langle p\rangle_{n}$, откуда, поскольку значение $\chi_{Q}(p)$ равно \pm 1 , следует соотношение

$$
\Psi^{n, m}\left(\langle p\rangle_{n}\right)=\chi_{Q}(p)^{n} p^{m(m-n) / 2}\langle p\rangle_{m} .
$$

По индукции из формул (4.16) и (4.17) легко следует, что для $2 \leqslant n<m$ выполняются соотношения

$$
\Psi^{m, n}\left(T_{m-1}^{m}\left(p^{2}\right)\right)=(a p)^{m-n} T_{n-1}^{n}\left(p^{2}\right)+a^{m-n-1}\left(\sum_{i=0}^{m-n-1} p^{i} b_{m-i}(p)\right)\langle p\rangle_{n},
$$

где $a=\chi_{Q}(p) p^{-m / 2}$. Из этих соотношений и соотношения (4.21) следует, что можно взять

$$
\begin{aligned}
\Psi^{n, m}\left(T_{n-1}^{n}\left(p^{2}\right)\right)= & \chi_{Q}(p)^{n} p^{(m-n)(m-2) / 2} T_{m-1}^{m}\left(p^{2}\right) \\
& \quad-\chi_{Q}(p)^{n+1} p^{\left(m^{2}-m n-m+2 n\right) / 2}\left(\sum_{i=0}^{m-n-1} p^{i} b_{m-i}(p)\right)\langle p\rangle_{m}
\end{aligned}
$$

(отметим, что $\chi_{Q}(p)= \pm 1$ и $m$ четно). Из этих формул, в частности, получаем соотношение

$$
\begin{aligned}
& \Psi^{2,4}\left(T_{1}^{2}\left(p^{2}\right)\right)=p^{2} T_{3}^{4}\left(p^{2}\right)-\chi_{Q}(p) p^{4}\left(b_{4}(p)+p b_{3}(p)\right)\langle p\rangle_{4} \\
& \quad=p^{2} T_{3}^{4}\left(p^{2}\right)-\chi_{Q}(p) p^{2}\left(p^{6}+p^{5}+p^{3}+p^{2}+\chi_{Q}(p)\left(p^{2}-1\right)\right)\langle p\rangle_{4} .
\end{aligned}
$$

Обратимся теперь к формулам для действия на тета-суммы операторов Гекке, отвечающих коэффициентам спинорных $p$-многочленов

$$
\mathbf{Q}_{p}^{n}(t)=\sum_{j=0}^{2^{n}}(-1)^{j} \mathbf{q}_{j}^{n}(p) t^{j}
$$


над $p$-подкольцами колец $\mathcal{H}_{0}^{n}(q)$ для простых чисел $p$, не делящих ступень $q$ (см., например, [9; (3.3.78)]). Эти многочлены представляют значительный интерес, поскольку, подставляя $t=\psi(p) p^{-s}$ с подходящим характером Дирихле $\psi$ и заменяя коэффициенты $\mathbf{q}_{j}^{n}(p)$ собственными членами $\Lambda\left(\mathbf{q}_{j}^{n}(p)\right)$ соответствующих операторов Гекке, действующих на некоторую собственную функцию $F \in \mathfrak{M}_{w}^{n}(q, \chi)$, мы получаем знаменатели

$$
\mathbf{Q}_{p}\left(\psi(p) p^{-s}, \Lambda\right)=\sum_{j=0}^{2^{n}}(-1)^{j} \Lambda\left(\mathbf{q}_{j}^{n}(p)\right) p^{-s j}
$$

p-множителей регулярной дзета-функции

$$
Z_{F}(s, \psi)=\prod_{p \nmid q} \mathbf{Q}_{p}\left(\psi(p) p^{-s}, \Lambda\right)^{-1}
$$

с характером $\psi$, отвечающей выбранной собственной функции. В случае $n=$ 1 - это дзета-функция Гекке эллиптической модулярной формы $F$; для $n=2$ указанное произведение определяет дзета-функцию Андрианова собственной функции $F$ рода 2. Отметим, что вопросы диагонализации регулярных операторов Гекке на пространствах зигелевых модулярных форм рассмотрены, например, в книге [9; $\$ 4.1 .2]$. Диагонализации сингулярных операторов Гекке посвящены, в частности, работы [16]-[20].

Мы ограничимся рассмотрением действия на тета-суммы операторов Гекке, отвечающих коэффициентам $\mathbf{q}_{1}^{n}(p), \mathbf{q}_{2^{n}-1}^{n}(p), \mathbf{q}_{2^{n}}^{n}(p)$ и $\mathbf{q}_{2}^{2}(p)$, поскольку этого будет достаточно для вычисления эйлеровых произведений (4.23), отвечающих собственным функциям рода $n=1$ и 2. Согласно формулам, полученным в [9; (3.3.81), (3.3.79), (3.3.80) и упражнение 3.3.38], в приведенных выше обозначениях эти коэффициенты могут быть записаны в виде

$$
\begin{aligned}
\mathbf{q}_{1}^{n}(p) & =T^{n}(p), \\
\mathbf{q}_{2^{n}-1}(p) & =\left(p^{n(n+1) / 2}\langle p\rangle_{n}\right)^{2^{n-1}-1} T^{n}(p), \\
\mathbf{q}_{2^{n}}^{n}(p) & =\left(p^{n(n+1) / 2} T_{n}^{n}\left(p^{2}\right)\right)^{2^{n-1}}=\left(p^{n(n+1) / 2}\langle p\rangle_{n}\right)^{2^{n-1}}, \\
\mathbf{q}_{2}^{2}(p) & =p T_{1}^{2}\left(p^{2}\right)+p\left(p^{2}+1\right)\langle p\rangle_{2} .
\end{aligned}
$$

Так что, в частности, мы имеем формулы

$$
\begin{aligned}
\mathbf{Q}_{p}^{1}(t)= & \langle 1\rangle_{1}-T^{1}(p) t+p\langle p\rangle_{1} t^{2} \\
\mathbf{Q}_{p}^{2}(t)= & \langle 1\rangle_{2}-T^{2}(p) t+\left(p T_{1}^{2}\left(p^{2}\right)+p\left(p^{2}+1\right)\langle p\rangle_{2}\right) t^{2} \\
& \quad-p^{3}\langle p\rangle_{2} T^{2}(p) t^{3}+p^{6}\langle p\rangle_{2}^{2} t^{4}
\end{aligned}
$$

Отсюда следует, что достаточно рассмотреть действие операторов, отвечающих элементам $T^{n}(p),\langle p\rangle_{n}$ и $T_{1}^{2}\left(p^{2}\right)$. Согласно (4.14), (4.10), (4.20) и (2.29), мы получаем

$$
\Theta(Z ; \mathbf{P},\langle Q\rangle) \mid T^{n}(p)=\delta_{n}^{m}(p) \Theta(Z ; \mathbf{P} \mid \boldsymbol{\tau}(p),\langle Q\rangle)
$$


где, исключая случаи, когда $n \leqslant m / 2-1$ и $\chi_{Q}(p)=-1$, коэффициент задается формулами

$$
\begin{aligned}
\delta_{n}^{m}(p) & =p^{-n k+m / 2} \gamma_{n}^{m}(p)^{-1} \prod_{j=1}^{m / 2}\left(1+\chi_{Q}(p) p^{-j}\right) \\
& =\chi_{Q}(p)^{n} p^{-n\left(k+\frac{m}{2}\right)+\frac{n(n+1)}{2}} \times \begin{cases}\prod_{j=1}^{\frac{m}{2}-n}\left(1+\chi_{Q}(p) p^{j-1}\right)^{-1}, & \text { если } n<m / 2 \\
1, & \text { и } \chi_{Q}(p) \neq-1, \\
\prod_{j=1}^{n-\frac{m}{2}}\left(1+\chi_{Q}(p) p^{-i}\right), & \text { если } n=m / 2,\end{cases}
\end{aligned}
$$

далее, согласно соотношениям (4.14), (4.12) и (4.21), мы получаем

$$
\Theta(Z ; \mathbf{P},\langle Q\rangle) \mid\langle p\rangle_{n}=\chi_{Q}(p)^{n} p^{-n(m / 2+k)} \Theta\left(Z ; \mathbf{P} \mid[p]_{m},\langle Q\rangle\right) ;
$$

наконец, если $m=4$, то из соотношений $(4.14),(4.11),(4.22)$ и (4.27) следует формула

$$
\begin{gathered}
\Theta(Z ; \mathbf{P},\langle Q\rangle) \mid T_{1}^{2}\left(p^{2}\right)=\chi_{Q}(p) p^{-(3+4 k)} \theta\left(Z ; \mathbf{P} \mid \boldsymbol{\tau}\left[1, p, p, p^{2}\right],\langle Q\rangle\right) \\
\quad+p^{2-4 k}\left(\alpha_{4}(p)-\chi_{Q}(p) p^{-6}\left(b_{4}(p)+p b_{3}(p)\right)\right) \theta\left(Z ; \mathbf{P} \mid[p]_{4}, Q\right) \\
=\chi_{Q}(p) p^{-(3+4 k)} \theta\left(Z ; \mathbf{P} \mid \boldsymbol{\tau}\left[1, p, p, p^{2}\right],\langle Q\rangle\right) \\
\quad+p^{-4-4 k}\left(\chi_{Q}(p) p^{2}-1\right) \theta\left(Z ; \mathbf{P} \mid[p]_{4},\langle Q\rangle\right) .
\end{gathered}
$$

Пусть $Q$ - некоторая четная положительно-определенная матрица ступени $q$ и четного порядка $m=2 k$, и пусть $Q_{1}, \ldots, Q_{h}$ - некоторая система представителей из класса эквивалентности (2.14), содержащихся в классе подобия (2.13) матрицы $Q$. Предположим, что порядки групп собственных единиц матриц $Q_{i}$ равны друг другу. Далее, пусть

$$
\mathbf{P}=\left(P_{1}, \ldots, P_{h}\right) \in \mathcal{I}_{k}^{n}\langle Q\rangle
$$

- некоторый инвариантый гармонический вектор вида (3.11) рода $n$ и веса $k$ относительно системы $Q_{1}, \ldots, Q_{h}$. Так как, по следствию 2.7, элементы $\boldsymbol{\tau}(p)$ с простыми $p \nmid q$ коммутируют друг с другом и по лемме 2.5 они удовлетворяют соотношениям (2.31), то по предложению 3.5 каждое из инвариантных подпространств пространства $\mathcal{I}_{k}^{n}\langle Q\rangle$ имеет базис, состоящий из общих собственных векторов всех операторов $\boldsymbol{\tau}(p)$. Если вектор $\mathbf{P}$ из пространства $\mathcal{I}_{k}^{n}\langle Q\rangle$ является собственным вектором с собственными числами $\lambda(\boldsymbol{\tau}(p))$, то на основании формулы (4.26) тета-сумма $\Theta(Z ; \mathbf{P}, Q)$ является собственной функцией оператора Гекке $\mid T(p)$,

$$
\Theta(Z ; \mathbf{P},\langle Q\rangle) \mid T(p)=\Lambda(T(p)) \Theta(Z ; \mathbf{P},\langle Q\rangle),
$$

с собственным числом

$$
\Lambda(T(p))=\delta_{n}^{m}(p) \lambda(\boldsymbol{\tau}(p)),
$$

кроме тех случаев, когда $n \leqslant m / 2-1$ и $\chi_{Q}(p)=-1$. 
Теперь мы рассмотрим связи дзета-функций (2.47), отвечающих собственным векторам колец классов автоморфов бинарных и кватернарных квадратичных форм на пространствах гармонических векторов, с дзета-функциями (4.23) тета-сумм, соответствующими этим гармоническим векторам. Отметим, что для матриц $Q$ порядков $m=2$ или 4 в предположении, что кольцо классов автоморфов $\mathcal{H}\langle Q\rangle$ является ручным, все операторы Гекке, отвечающие коэффициентам локальных дзета-рядов (2.41) для всех простых чисел $p$, не делящих $\operatorname{det} Q$, могут быть одновременно диагонализированы на каждом инвариантном подпространстве гармонических векторов.

Пусть $Q$ - матрица положительно-определенной бинарной квадратичной формы, пусть род $n=1$, и пусть $\mathbf{P} \in \mathcal{I}_{k}^{1}\langle Q\rangle$ - некоторый собственный вектор всех операторов Гекке $\mid \boldsymbol{\tau}(p)$ с простыми числами $p$, не делящими ступень $q$ матрицы $Q$. По предположению и условию (3.4) для этих простых $p$ имеют место соотношения

$$
\mathbf{P} \mid \boldsymbol{\tau}(p)=\lambda(\boldsymbol{\tau}(p)) \mathbf{P} \quad \text { и } \quad \mathbf{P} \mid[p]=\mathbf{P}(p X)=p^{k} \mathbf{P}=\lambda([p]) \mathbf{P} .
$$

Отсюда следует, что $p$-множитель (2.46) дзета-функции (2.47), отвечающий вектору $\mathbf{P}$, равен

$$
Z_{p}\left(p^{-s}, \mathbf{P}\right)=\left(1-\lambda(\tau(p)) p^{-s}+\chi_{Q}(p) \lambda([p]) p^{-2 s}\right)^{-1} .
$$

С другой стороны, согласно соотношениям (4.29) и (4.27), тета-ряд

$$
\Theta(z ; \mathbf{P}, Q) \in \mathfrak{M}_{1+k}^{1}\left(q, \chi_{Q}\right)
$$

является собственной функцией операторов Гекке $|T(p)=| T^{1}(p)$ и $|\langle p\rangle=|\langle p\rangle_{1}$ с собственными числами

$$
\Lambda(T(p))=\delta_{1}^{2}(p) \lambda(\boldsymbol{\tau}(p))=\chi_{Q}(p) p^{-k} \lambda(\boldsymbol{\tau}(p))
$$

и

$$
\Lambda(\langle p\rangle)=\chi_{Q}(p) p^{-(1+k)}=\chi_{Q}(p) p^{-(1+2 k)} \lambda([p])
$$

соответственно. Отсюда следует, что для $p$-множителя дзета-функции (4.23) с характером $\psi=\chi_{Q}$, отвечающей собственной функции $\Theta(z ; \mathbf{P}, Q)$, имеет место тождество

$$
\begin{aligned}
\mathbf{Q}_{p}^{1}\left(\chi_{Q}(p) p^{k-s}, F\right)^{-1} & =\left(1-\Lambda(T(p)) \chi_{Q}(p) p^{k-s}+\Lambda(\langle p\rangle) p\left(\chi_{Q}(p) p^{k-s}\right)^{2}\right)^{-1} \\
& =\left(1-\lambda(\tau(p)) p^{-s}+\chi_{Q}(p) \lambda([p]) p^{-2 s}\right)^{-1}=Z_{p}(t, \mathbf{P})
\end{aligned}
$$

Из равенства локальных дзета-функций для всех простых $p$, не делящих ступень матрицы $Q$, следует равенство соответствующих эйлеровых произведений.

ТЕОРемА 4.2. Пусть $Q$ - матрица положительно-определенной бинарной квадратичной формы, $q$ - ступень матрищъ $Q$ и $\chi_{Q}$ - соответствующий характер Дирихле по модулю $q$, и пусть $\mathbf{P} \in \mathcal{I}_{k}^{1}\langle Q\rangle-$ гармонический собственный вектор для всех операторов Гекке $\mid \boldsymbol{\tau}(p)$ с простыми числами $p \nmid q$. Тогда тета-сумма рода 1 класса подобия матрищы $Q$, отвечающая вектору $\mathbf{P}$,

$$
F=\Theta(z ; \mathbf{P}, Q) \in \mathfrak{M}_{1+k}^{1}\left(q, \chi_{Q}\right),
$$


является собственной функцией всех операторов Гекке $|T(p)=| T^{1}(p)$ и соответствующие регулярные дзета-функиии (2.47) и (4.23) связаны тождеством

$$
Z^{*}(s, \mathbf{P})=Z_{F}\left(s-k, \chi_{Q}\right) .
$$

Наконец, мы докажем, что аналогичные соотношения имеют место также в случае квадратичных форм от $m=4$ переменных, гармонических форм рода $n=2$ и соответствующих тета-рядов.

ТеОрема 4.3. Пусть $Q$ - матрица положительно-определенной кватернарной квадратичной формы, $q$ - ступень матрицы $Q$ и $\chi_{Q}$ - соответствующий характер Дирихле по модулю $q$, и пусть $\mathbf{P} \in \mathcal{I}_{k}^{2}\langle Q\rangle-$ гармонический собственный вектор для всех операторов Гекке $|\boldsymbol{\tau}(p) u| \boldsymbol{\tau}\left[1, p, p, p^{2}\right]$ с простыми числами $p \nmid q$. Тогда тета-сумма рода 2 класса подобия матриць $Q$, отвечающая вектору $\mathbf{P}$,

$$
F=\Theta(Z ; \mathbf{P}, Q) \in \mathfrak{M}_{2+k}^{2}\left(q, \chi_{Q}\right),
$$

является собственной функиией всех операторов Гекке вида $|T(p)=| T^{2}(p)$ u $\mid T_{1}^{2}\left(p^{2}\right)$ с простыми числами $p \nmid q$ и соответствующие регулярные дзетафункиии (2.47) и (4.23) связаны тождеством

$$
Z^{*}(s, \mathbf{P})=Z_{F}\left(s-2 k-1, \chi_{1} / q\right),
$$

где $\chi_{1} / q$ обозначает единичный характер по модулю $q$.

ДокАЗАТЕЛЬство. По предположению, для каждого простого числа $p$, не делящего ступень $q$, имеют место соотношения

$$
\mathbf{P}|\boldsymbol{\tau}(p)=\lambda(\boldsymbol{\tau}(p)) \mathbf{P}, \quad \mathbf{P}| \boldsymbol{\tau}\left[1, p, p, p^{2}\right]=\lambda\left(\boldsymbol{\tau}\left[1, p, p, p^{2}\right]\right) \mathbf{P} .
$$

Используя свойство (3.4), получаем

$$
\mathbf{P} \mid[p]_{4}=\mathbf{P}(p X)=p^{2 k} \mathbf{P}=\lambda\left([p]_{4}\right) \mathbf{P} .
$$

Отсюда, согласно формулам (2.45) и (2.42), следует, что $p$-множитель (2.46) ортогональной дзета-функции (2.47), отвечающей собственному вектору $\mathbf{P}$, равен

$$
\begin{gathered}
Z_{p}(t, \mathbf{P})=\left(1-\lambda(\boldsymbol{\tau}(p)) p^{-s}+\left(\chi_{Q}(p) \lambda\left(\boldsymbol{\tau}\left[1, p, p, p^{2}\right]\right)+\left(1+\chi_{Q}(p)\right) p \lambda\left([p]_{2}\right)\right) p^{-2 s}\right. \\
\left.-p \lambda\left([p]_{2}\right) \lambda(\boldsymbol{\tau}(p)) p^{-3 s}+p^{2} \lambda\left([p]_{2}\right)^{2} p^{-4 s}\right)^{-1} .
\end{gathered}
$$

С другой стороны, по формулам (4.29), (4.30), (4.27) и (4.28) соответственно, получаем соотношения

$$
\begin{aligned}
\Theta(Z ; \mathbf{P},\langle Q\rangle) \mid T^{2}(p) & =\delta_{2}^{4}(p) \lambda(\boldsymbol{\tau}(p)) \Theta(Z ; \mathbf{P},\langle Q\rangle)=p^{-2 k-1} \lambda(\boldsymbol{\tau}(p)) \Theta(Z ; \mathbf{P},\langle Q\rangle), \\
\Theta(Z ; \mathbf{P},\langle Q\rangle) \mid\langle p\rangle_{2} & =\chi_{Q}(p)^{2} p^{-2(k+2)} \Theta\left(Z ; \mathbf{P} \mid[p]_{4},\langle Q\rangle\right) \\
& =p^{-4 k-4} \lambda\left([p]_{4}\right) \Theta(Z ; \mathbf{P},\langle Q\rangle)
\end{aligned}
$$

и

$$
\begin{aligned}
\Theta(Z ; \mathbf{P},\langle Q\rangle) \mid T_{1}^{2}\left(p^{2}\right)= & \chi_{Q}(p) p^{-(4 k+3)} \Theta\left(Z ; \mathbf{P} \mid \boldsymbol{\tau}\left[1, p, p, p^{2}\right],\langle Q\rangle\right) \\
& +p^{-4 k-4}\left(\chi_{Q}(p) p^{2}-1\right) \Theta\left(Z ; \mathbf{P} \mid[p]_{4},\langle Q\rangle\right) \\
= & \left(\chi_{Q}(p) p^{-(4 k+3)} \lambda\left(\boldsymbol{\tau}\left[1, p, p, p^{2}\right]\right)\right. \\
& \left.+p^{-4 k-4}\left(\chi_{Q}(p) p^{2}-1\right) \lambda\left([p]_{4}\right)\right) \Theta(Z ; \mathbf{P},\langle Q\rangle) .
\end{aligned}
$$


Откуда по второй формуле (4.25) мы заключаем, что $p$-множитель дзета-функции (4.23), соответствующей собственной функции $F=\Theta(Z ; \mathbf{P}, Q)$ операторов Гекке $\mid T$ из кольца $\mathcal{H}_{0}^{2}(q)$ с $s-2 k-1$ вместо $s$ и $\psi=\chi_{1} / q$, имеет вид

$$
\begin{aligned}
\mathbf{Q}_{p}^{2}\left(p^{2 k+1-s}, \Lambda\right)^{-1}=( & -\Lambda\left(T^{2}(p)\right) t+\Lambda\left(p T_{1}^{2}\left(p^{2}\right)+p\left(p^{2}+1\right)\langle p\rangle_{2}\right) t^{2} \\
& \left.-\Lambda\left(p^{3}\langle p\rangle_{2} T^{2}(p)\right) t^{3}+p^{6} \Lambda\left(\langle p\rangle_{2}^{2}\right) t^{4}\right)^{-1}
\end{aligned}
$$

где $\Lambda(T)$ - соответствующие собственные числа и $t=p^{2 k+1-s}$, что можно переписать в виде

$$
\begin{gathered}
\left(1-p^{-2 k-1} \lambda(\boldsymbol{\tau}(p)) t+\left(p \left(\chi_{Q}(p) p^{-(4 k+3)} \lambda\left(\boldsymbol{\tau}\left[1, p, p, p^{2}\right]\right)\right.\right.\right. \\
\left.\left.+p^{-4 k-4} p\left(\chi_{Q}(p) p^{2}-1\right) \lambda\left([p]_{4}\right)\right)+p\left(p^{2}+1\right) p^{-4 k-4} \lambda\left([p]_{4}\right)\right) t^{2} \\
\left.+p^{3} p^{-4 k-4} \lambda\left([p]_{4}\right) p^{-2 k-1} \lambda(\boldsymbol{\tau}(p)) t^{3}+p^{6} p^{-8 k-8} \lambda\left([p]_{4}\right)^{2} t^{4}\right)^{-1} \\
=\left(1-\lambda(\boldsymbol{\tau}(p)) p^{-s}+\left(\chi_{Q}(p) \lambda\left(\boldsymbol{\tau}\left[1, p, p, p^{2}\right]\right)+\left(1+\chi_{Q}(p)\right) p \lambda\left([p]_{4}\right)\right) p^{-2 s}\right. \\
\left.-p \lambda\left([p]_{4}\right) \lambda(\boldsymbol{\tau}(p)) p^{-3 s}+p^{2} \lambda\left([p]_{4}\right)^{2} p^{-4 s}\right)^{-1}=Z_{p}(t, \mathbf{P}) .
\end{gathered}
$$

Равенства локальных дзета-функций для всех простых $p$, не делящих ступень матрицы $Q$, доказывают равенство эйлеровых произведений.

\section{§ 5. Бинарные формы фундаментальных дискриминантов}

В этом параграфе мы докажем, что в случае бинарных квадратичных форм фундаментальных дискриминантов соответствующие глобальные дзета-функции фактически совпадают с дзета-функциями соответствующих квадратичных полей с подходящими характерами Гекке. Получающиеся тождества можно рассматривать как естественные расширения тождеств теоремы 4.2 на эйлеровы произведения по всем, а не только регулярным, простым числам.

Пусть

$$
Q_{1}=\left(\begin{array}{cc}
2 a_{1} & b_{1} \\
b_{1} & 2 c_{1}
\end{array}\right), \ldots, Q_{h}=\left(\begin{array}{cc}
2 a_{h} & b_{h} \\
b_{h} & 2 c_{h}
\end{array}\right)
$$

- матрицы некоторой системы представителей

$$
\mathbf{q}_{1}(X)=a_{1} x_{1}^{2}+b_{1} x_{1} x_{2}+c_{1} x_{2}^{2}, \ldots, \mathbf{q}_{h}(X)=a_{h} x_{1}^{2}+b_{h} x_{1} x_{2}+c_{h} x_{2}^{2}
$$

из классов собственной эквивалентности целочисленных положительно-определенных бинарных квадратичных форм делителя $\delta=\operatorname{gcd}\left(a_{i}, b_{i}, c_{i}\right)=1$ и $\partial и с$ $к р и м и н а н т а ~ b_{i}^{2}-4 a_{i} c_{i}=-\operatorname{det} Q_{i}=-d<0$. Отметим, что в этом случае все матрицы $Q_{i}$ имеют одинаковую ступень $q=d$.

С другой стороны, рассмотрим мнимое квадратичное поле $K=\mathbb{Q}(\sqrt{-d})$. Пусть $\Delta<0$ будет дискриминант поля $K$, тогда $K=\mathbb{Q}(\sqrt{\Delta})$ и $-d$ имеет вид $\Delta t^{2}$ с $t \in \mathbb{N}$. Для упрощения предстоящих рассмотрений мы будем предполагать, что дискриминант форм (5.1) является фундаментальным, т.е. совпадает с дискриминантом поля $K$, так что $\Delta=-d$. 
Если

$$
\mathbf{q}_{i}(X)=a_{i}\left(x_{1}-\gamma_{i} x_{2}\right)\left(x_{1}-\bar{\gamma}_{i} x_{2}\right), \quad \text { где } \quad \gamma_{i}=\frac{-b_{i}+\sqrt{-d}}{2 a_{i}} \in \mathbb{H}_{1},
$$

- стандартное разложение формы $\mathbf{q}_{i}$, мы будем говорить, что число $\gamma_{i}$ является корнем формы $\mathbf{q}_{i}$. Все корни $\gamma_{i}$ содержатся в поле $K$. Мы ассоциируем с каждой из форм $\mathbf{q}_{i}($ полный $) \mathbb{Z}$-модуль ранга 2 в поле $K$ вида

$$
\mathcal{M}_{i}=\left\{1, \gamma_{i}\right\}=\left\{\mathbb{Z}+\gamma_{i} \mathbb{Z}\right\} .
$$

Норма $N\left(\mathcal{M}_{i}\right)$ модуля $\mathcal{M}_{i}$ равна $1 / a_{i}$, а его кольцо множителей,

$$
\mathcal{O}\left(\mathcal{M}_{i}\right)=\left\{\alpha \in K \mid \alpha \mathcal{M}_{i} \subset \mathcal{M}_{i}\right\}=\left\{1, a_{i} \gamma_{i}\right\}=\{\alpha \in K \mid \alpha+\bar{\alpha}, \alpha \bar{\alpha} \in \mathbb{Z}\}=\mathcal{O},
$$

совпадает с кольцом целых чисел поля $K$. Модули $\mathcal{M}_{1}, \ldots, \mathcal{M}_{h}$ образуют полную систему представителей из классов эквивалентных полных модулей в поле $K$ с кольцом множителей $\mathcal{O}$. По поводу деталей о модулях в квадратичных полях и их связях с бинарными квадратичными формами см., например, [9; приложение 3].

Целочисленная матрица $D=\left(\begin{array}{ll}a & b \\ c & d\end{array}\right)$ с $\operatorname{det} D>0$ является (собственным) автоморфом матрицы $Q_{i}$ в $Q_{j}$ с мультипликатором $\mu$ в том и только том случае, если $\mathbf{q}_{i}(D X)=\mu \mathbf{q}_{j}$, т.е.

$$
\begin{aligned}
a_{i}\left(a x_{1}\right. & \left.+b x_{2}-\gamma_{i}\left(c x_{1}+d x_{2}\right)\right)\left(a x_{1}+b x_{2}-\bar{\gamma}_{i}\left(c x_{1}+d x_{2}\right)\right) \\
= & a_{i}\left(\left(a-\gamma_{i} c\right) x_{1}-\left(\gamma_{i} d-b\right) x_{2}\right)\left(\left(a-\bar{\gamma}_{i} c\right) x_{1}-\left(\bar{\gamma}_{i} d-b\right) x_{2}\right) \\
= & a_{i} N\left(a-\gamma_{i} c\right)\left(x_{1}-\frac{\gamma_{i} d-b}{a-\gamma_{i} c} x_{2}\right)\left(x_{1}-\frac{\bar{\gamma}_{i} d-b}{a-\bar{\gamma}_{i} c} x_{2}\right) \\
= & \mu a_{j}\left(x_{1}-\gamma_{j} x_{2}\right)\left(x_{1}-\bar{\gamma}_{j} x_{2}\right),
\end{aligned}
$$

где $N(\alpha)=\alpha \bar{\alpha}$ обозначает норму числа $\alpha \in K$. Так как оба числа $\gamma_{j}$ и $\frac{\gamma_{i} d-b}{a-\gamma_{i} c}$ принадлежат верхней полуплоскости, последнее тождество эквивалентно соотношениям

$$
\frac{1}{N\left(\mathcal{M}_{i}\right)} N\left(a-\gamma_{i} c\right)=\frac{\mu}{N\left(\mathcal{M}_{j}\right)} \quad \text { и } \quad \frac{\gamma_{i} d-b}{a-\gamma_{i} c}=\gamma_{j} .
$$

Последнее соотношение означает, что второй столбец матрицы $D$ однозначно определяется ее первым столбцом, а первый столбец удовлетворяет условию $\left(a-\gamma_{i} c\right) \gamma_{j} \in \mathcal{M}_{i}$, т.е. условию $\left(a-\gamma_{i} c\right) \mathcal{M}_{j} \subset \mathcal{M}_{i}$, которое можно записать как включение $a-\gamma_{i} c \in \mathcal{M}_{i} \mathcal{M}_{j}^{-1}$. Таким образом, целочисленная матрица $D=\left(\begin{array}{ll}a & b \\ c & d\end{array}\right)$ с $\operatorname{det} D>0$ является собственным автоморфом матрицы $Q_{i}$ в $Q_{j}$ с мультипликатором $\mu$ в том и только том случае, если

$$
a-\gamma_{i} c \in \mathcal{M}_{i} \mathcal{M}_{j}^{-1} \quad \text { и } \quad N\left(a-\gamma_{i} c\right)=\mu N\left(\mathcal{M}_{i}\right) / N\left(\mathcal{M}_{j}\right)=\mu N\left(\mathcal{M}_{i} \mathcal{M}_{j}^{-1}\right) .
$$

Сопоставим каждому автоморфу

$$
D=\left(\begin{array}{ll}
a & b \\
c & d
\end{array}\right) \in A_{i j}(\mu)=R^{+}\left(Q_{i}, \mu Q_{j}\right)
$$


число

$$
\alpha(D)=\alpha_{i j}(D)=a-\gamma_{i} c \in \mathcal{M}_{i} \mathcal{M}_{j}^{-1} .
$$

Как мы видели выше, отображение $D \mapsto \alpha(D)$ определяет взаимно однозначное соответствие между множеством $A_{i j}(\mu)$ и множеством всех чисел $\alpha \in \mathcal{M}_{i} \mathcal{M}_{j}^{-1}$ с $N(\alpha)=\mu$. В частности, оно является взаимно однозначным отображением каждой из групп единиц $\mathbf{E}_{i}=A_{i i}(1)$ на группу единиц $\mathcal{E}=E(\mathcal{O})$ кольца $\mathcal{O}$, откуда

$$
\#\left(\mathbf{E}_{1}\right)=\cdots=\#\left(\mathbf{E}_{h}\right)=\#(E(\mathcal{O}))
$$

Если $D=\left(\begin{array}{ll}a & b \\ c & d\end{array}\right) \in A_{i j}(\mu)$ и $D^{\prime}=\left(\begin{array}{ll}a^{\prime} & b^{\prime} \\ c^{\prime} & d^{\prime}\end{array}\right) \in A_{j k}(\nu)$, то произведение

$$
D D^{\prime}=\left(\begin{array}{ll}
a^{\prime \prime} & b^{\prime \prime} \\
c^{\prime \prime} & d^{\prime \prime}
\end{array}\right)
$$

где $a^{\prime \prime}=a a^{\prime}+b c^{\prime}$ и $c^{\prime \prime}=c a^{\prime}+d c^{\prime}$, содержится в $A_{i k}(\mu \nu)$ и, используя соотношения (5.3), мы получаем

$$
\begin{aligned}
\alpha\left(D D^{\prime}\right) & =\alpha_{i k}\left(D D^{\prime}\right)=a^{\prime \prime}-\gamma_{i} c^{\prime \prime}=a a^{\prime}+b c^{\prime}-\gamma_{i}\left(c a^{\prime}+d c^{\prime}\right) \\
& =\left(a-\gamma_{i} c\right) a^{\prime}-\left(\gamma_{i} d-b\right) c^{\prime}=\left(a-\gamma_{i} c\right)\left(a^{\prime}-\frac{\gamma_{i} d-b}{a-\gamma_{i} c} c^{\prime}\right)=\alpha_{i j}(D) \alpha_{j k}\left(D^{\prime}\right) .
\end{aligned}
$$

Отсюда следует, что каждый двойной класс $\mathbf{E}_{i} D \mathbf{E}_{j} \subset \mathbf{A}_{i j}$ совпадает с левым классом $\mathbf{E}_{i} D$, и главные модули (идеалы) $(\alpha(D))=\alpha(D) \mathcal{O}$ кольца $\mathcal{O}$ находятся во взаимно однозначном соответствии с этими двойными или левыми классами. Это соответствие, которое мы также будем обозначать буквой $\alpha$, так что $\alpha\left(\mathbf{E}_{i} D \mathbf{E}_{j}\right)=\alpha\left(\mathbf{E}_{i} D\right)=\alpha(D) \mathcal{O}$, очевидно, совместимо с умножением двойных классов в кольце классов автоморфов $\mathcal{H}=\mathcal{H}\langle Q\rangle$ и обычным умножением модулей с кольцом множителей $\mathcal{O}$. Кроме того,

$$
N(\alpha(D) \mathcal{O})=N(\alpha(D))=\mu(D),
$$

где $N$ обозначает норму модуля и числа соответственно. Следовательно, $\mathbb{C}$-линейное расширение $\boldsymbol{\alpha}$ соответствия $\alpha$ на расширенное кольцо Гекке-Шимуры

$$
\widetilde{\mathcal{H}}=\mathcal{H}_{\mathbb{C}}\left(Q_{1}, \ldots, Q_{h}\right)=\mathcal{H}\left(Q_{1}, \ldots, Q_{h}\right) \otimes \mathbb{C},
$$

где $\otimes$ обозначает тензорное произведение над $\mathbb{Z}$ (расширение кольца скаляров), рассматриваемое над полем $\mathbb{C}$, является изоморфизмом кольца $\widetilde{\mathcal{H}}$ на кольцо $\mathbf{M}(\mathcal{O})$ всех матриц порядка $h,\{i, j\}$-элементы которых являются формальными конечными линейными комбинациями с коэффициентами из $\mathbb{C}$ ненулевых главных модулей $\alpha \mathcal{O}$ с $\alpha \in \mathcal{M}_{i} \mathcal{M}_{j}^{-1}$ (мы расширяем кольцо скаляров $\mathbb{Z}$ до $\mathbb{C}$, поскольку ниже потребуется использовать комплексные коэффициенты).

Найдем $\boldsymbol{\alpha}$-образы элементов $\boldsymbol{\tau}(\mu)=\left(\tau_{i j}(\mu)\right)$ вида (2.29). По определению мы получаем

$$
\boldsymbol{\alpha}(\boldsymbol{\tau}(\mu))=\left(\boldsymbol{\alpha}_{i j}(\mu)\right), \quad \text { где } \quad \boldsymbol{\alpha}_{i j}(\mu)=\alpha\left(\tau_{i j}(\mu)\right)=\sum_{\substack{(\alpha) \subset \mathcal{M}_{i} \mathcal{M}_{j}^{-1}, N(\alpha)=\mu N\left(\mathcal{M}_{i} \mathcal{M}_{j}^{-1}\right)}}(\alpha) .
$$


Условия $(\alpha) \subset \mathcal{M}_{i} \mathcal{M}_{j}^{-1}$ и $N(\alpha)=\mu N\left(\mathcal{M}_{i} \mathcal{M}_{j}^{-1}\right)$ означают, что главный модуль $(\alpha)$ имеет вид $\mathfrak{A} \mathcal{M}_{i} \mathcal{M}_{j}^{-1}$, где $\mathfrak{A}-$ некоторый идеал кольца $\mathcal{O}$ (т.е. полный модуль с кольцом множителей $\mathcal{O}$, содержащийся в $\mathcal{O})$ с нормой $N(\mathfrak{A})=\mu$ и такой, что модуль $\mathfrak{A} \mathcal{M}_{i} \mathcal{M}_{j}^{-1}$ является главным. Таким образом,

$$
\boldsymbol{\alpha}_{i j}(\mu)=\sum_{\substack{\mathfrak{A} \subset \mathcal{O}, N(\mathfrak{A})=\mu \\ \mathcal{M}_{i} \mathcal{M}_{j}^{-1} \mathfrak{A} \sim \mathcal{O}}} \mathfrak{A} \mathcal{M}_{i} \mathcal{M}_{j}^{-1}
$$

Обозначим через $\mathrm{Cl}(K)$ группу классов эквивалентных полных модулей поля $K$, т.е. факторгруппу мультипликативной группы всех полных модулей по подгруппе главных модулей, и пусть $\widehat{\mathrm{Cl}}(K)$ обозначает группу характеров группы $\mathrm{Cl}(K)$. По соотношениям ортогональности для характеров мы получаем

$$
\begin{aligned}
& \frac{1}{h} \sum_{\chi \in \widehat{\mathrm{Cl}}(K)}\left(\sum_{\mathfrak{A} \subset \mathcal{O}, N(\mathfrak{A})=\mu} \chi(\mathfrak{A}) \mathfrak{A}\right) \chi\left(\mathcal{M}_{i} \mathcal{M}_{j}^{-1}\right) \mathcal{M}_{i} \mathcal{M}_{j}^{-1} \\
& \quad=\sum_{\mathfrak{A} \subset \mathcal{O}, N(\mathfrak{A})=\mu} \frac{1}{h}\left(\sum_{\chi \in \widehat{\mathrm{Cl}}(K)} \chi\left(\mathfrak{A} \mathcal{M}_{i} \mathcal{M}_{j}^{-1}\right)\right) \mathfrak{A M}_{i} \mathcal{M}_{j}^{-1}=\boldsymbol{\alpha}_{i j}(\mu) .
\end{aligned}
$$

Поэтому мы можем переписать всю матрицу $\boldsymbol{\alpha}(\boldsymbol{\tau}(\mu))$ в виде

$$
\boldsymbol{\alpha}(\boldsymbol{\tau}(\mu))=\sum_{\chi \in \widehat{\mathrm{Cl}}(K)}\left(\sum_{\mathfrak{A} \subset \mathcal{O}, N(\mathfrak{A})=\mu} \chi(\mathfrak{A}) \mathfrak{A}\right) I(\chi),
$$

где

$$
I(\chi)=\frac{1}{h}\left(\chi\left(\mathcal{M}_{i} \mathcal{M}_{j}^{-1}\right) \mathcal{M}_{i} \mathcal{M}_{j}^{-1}\right) \subset \mathbf{M}(\mathcal{O}) .
$$

Сумма этих матриц

$$
\begin{aligned}
\sum_{\chi \in \widehat{\mathrm{Cl}}(K)} I(\chi) & =\frac{1}{h}\left(\sum_{\chi \in \widehat{\mathrm{Cl}}(K)} \chi\left(\mathcal{M}_{i} \mathcal{M}_{j}^{-1}\right) \mathcal{M}_{i} \mathcal{M}_{j}^{-1}\right) \\
& =\operatorname{diag}(\mathcal{O}, \ldots, \mathcal{O})=1_{\mathbf{M}(\mathcal{O})}=\mathbf{1}
\end{aligned}
$$

является единичным элементом кольца $\mathbf{M}(\mathcal{O})$. Кроме того, эти матрицы удовлетворяют идемпотентным соотношениям

$$
I(\chi) I\left(\chi^{\prime}\right)= \begin{cases}I(\chi), & \text { если } \chi=\chi^{\prime}, \\ \mathbf{0}, & \text { если } \chi \neq \chi^{\prime} .\end{cases}
$$

Действительно, имеют место равенства

$$
\begin{aligned}
I(\chi) I\left(\chi^{\prime}\right) & =\frac{1}{h^{2}}\left(\sum_{l=1}^{h} \chi\left(\mathcal{M}_{i} \mathcal{M}_{l}^{-1}\right) \mathcal{M}_{i} \mathcal{M}_{l}^{-1} \chi\left(\mathcal{M}_{l} \mathcal{M}_{j}^{-1}\right) \mathcal{M}_{l} \mathcal{M}_{j}^{-1}\right) \\
& =\frac{1}{h^{2}}\left(\chi\left(\mathcal{M}_{i}\right) \chi^{\prime}\left(\mathcal{M}_{j}^{-1}\right) \mathcal{M}_{i} \mathcal{M}_{j}^{-1} \sum_{l} \chi\left(\mathcal{M}_{l}^{-1}\right) \chi^{\prime}\left(\mathcal{M}_{l}\right)\right)
\end{aligned}
$$


и соотношения (5.12) следуют из формул

$$
\sum_{l} \chi\left(\mathcal{M}_{l}^{-1}\right) \chi^{\prime}\left(\mathcal{M}_{l}\right)=\sum_{l}\left(\chi^{\prime} \chi^{-1}\right)\left(\mathcal{M}_{l}\right)= \begin{cases}h, & \text { если } \chi=\chi^{\prime}, \\ 0, & \text { если } \chi \neq \chi^{\prime} .\end{cases}
$$

Сопоставим теперь каждому ненулевому идеалу $\mathfrak{A}$ кольца $\mathcal{O}$ матрицу $\{\mathfrak{A}\} \in$ $\mathbf{M}(\mathcal{O})$ вида

$$
\{\mathfrak{A}\}=\sum_{\chi \in \widehat{\mathrm{Cl}}(K)} \chi(\mathfrak{A}) \mathfrak{A} I(\chi)=\mathfrak{A} \sum_{\chi \in \widehat{\mathrm{Cl}}(K)} \chi(\mathfrak{A}) I(\chi) .
$$

В частности, по формуле (5.11) мы получаем, что матрица

$$
\{\mathcal{O}\}=\sum_{\chi \in \widehat{\mathrm{Cl}}(K)} \mathcal{O} I(\chi)=\mathcal{O} \cdot 1_{\mathbf{M}(\mathcal{O})}=\mathbf{1}
$$

является единичным элементом кольца $\mathbf{M}(\mathcal{O})$. Из формул (5.12) следуют соотношения

$$
\{\mathfrak{A}\}\{\mathfrak{B}\}=\sum_{\chi} \chi(\mathfrak{A}) \mathfrak{A} I(\chi) \sum_{\chi^{\prime}} \chi^{\prime}(\mathfrak{B}) \mathfrak{B} I\left(\chi^{\prime}\right)=\sum_{\chi} \chi(\mathfrak{A} \mathfrak{B}) \mathfrak{A} \mathfrak{B} I(\chi)=\{\mathfrak{A} \mathfrak{B}\} .
$$

Используя обозначения (5.13), мы можем переписать формулу (5.9) в виде

$$
\boldsymbol{\alpha}(\boldsymbol{\tau}(\mu))=\sum_{\mathfrak{A} \subset \mathcal{O}, N(\mathfrak{A})=\mu}\{\mathfrak{A}\} .
$$

Приведенные формулы позволяют записать $\boldsymbol{\alpha}$-образ формального ряда Дирихле (2.38) в терминах идеалов кольца $\mathcal{O}$ :

$$
\sum_{\mu=1}^{\infty} \frac{\boldsymbol{\tau}(\mu)}{\mu^{s}} \Rightarrow \sum_{\mu=1}^{\infty} \frac{\boldsymbol{\alpha}(\boldsymbol{\tau}(\mu))}{\mu^{s}}=\sum_{\mathfrak{A}} \frac{\{\mathfrak{A}\}}{N(\mathfrak{A})^{s}},
$$

где $\mathfrak{A}$ пробегает все ненулевые идеалы кольца $\mathcal{O}$. Это позволяет нам использовать мультипликативную теорию идеалов кольца $\mathcal{O}$ для "эйлерова разложения" указанных рядов. Используя формулу (5.14), мы можем записать рассматриваемый ряд в виде

$$
\prod_{\mathfrak{P}} \sum_{\delta=0}^{\infty} \frac{\{\mathfrak{A}\}^{\delta}}{N\{\mathfrak{P}\}^{\delta s}}=\prod_{\mathfrak{P}}\left(\mathbf{1}-\frac{\{\mathfrak{P}\}}{N(\mathfrak{P})^{s}}\right)^{-1}=\prod_{p} \prod_{\mathfrak{P} \mid p}\left(\mathbf{1}-\frac{\{\mathfrak{P}\}}{N(\mathfrak{P})^{s}}\right)^{-1}
$$

где $\mathfrak{P}$ пробегает все простые идеалы кольца $\mathcal{O}, p$ пробегает все рациональные простые числа, а в последнем произведении $\mathfrak{P}$ пробегает все простые идеалы, делящие идеал $(p)=p \mathcal{O}$.

Применим хорошо известные законы разложения простых чисел на простые идеалы кольца $\mathcal{O}$ (см., например, $\left[21 ;\right.$ гл. $3, \S 8$, теорема 2]). Пусть $\chi_{K}$ обозначает характер Дирихле, ассоциированный с полем $K$, и пусть $p$ - рациональное простое число. Тогда в случае $\chi_{K}(p)=1$ существуют ровно два сопряженных простых идеала $\mathfrak{P}$ и $\overline{\mathfrak{P}}$ кольца $\mathcal{O}$, делящих число $p$, эти идеалы удовлетворяют соотношениям $\mathfrak{P} \bar{P}=p \mathcal{O}$ и $N(\mathfrak{P})=N(\overline{\mathfrak{P}})=p$, так что, на 
основании формул (5.15) и (5.16) соответственно, мы получаем соотношения $\{\mathfrak{P}\}\{\overline{\mathfrak{P}}\}=p\{\mathcal{O}\}=p \mathbf{1}=p \boldsymbol{\alpha}(\boldsymbol{\tau}(1))$ и $\{\mathfrak{P}\}+\{\overline{\mathfrak{P}}\}=\boldsymbol{\alpha}(p)$; если $\chi_{K}(p)=-1$, то существует единственный простой идеал $\mathfrak{P}$, делящий число $p$, этот идеал удовлетворяет условиям $\mathfrak{P}=p \mathcal{O}$ и $N(\mathfrak{P})=p^{2}$, так что $\{\mathfrak{P}\}=\{p \mathcal{O}\}=\boldsymbol{\alpha}([p])$ и $\boldsymbol{\alpha}(\boldsymbol{\tau}(p))=0$; если же $\chi_{K}(p)=0$, то существует единственный простой идеал $\mathfrak{P}$, делящий число $p$, и этот идеал удовлетворяет условиям $\mathfrak{P}^{2}=p \mathcal{O}$ и $N(\mathfrak{P})=p$, так что $\{\mathfrak{P}\}=\boldsymbol{\alpha}(p)$. Отметим, что в рассматриваемой ситуации характер $\chi_{K}$ равен характеру $\chi_{Q}$ квадратичной формы с матрицей $Q$, эквивалентной любой из матриц $Q_{i}$. Поэтому эйлерово произведение может быть переписано в виде

$$
\sum_{\mu=1}^{\infty} \frac{\boldsymbol{\alpha}(\boldsymbol{\tau}(\mu))}{\mu^{s}}=\prod_{p \text { простые }}\left(\boldsymbol{\alpha}([1])-\frac{\boldsymbol{\alpha}(\boldsymbol{\tau}(p))}{p^{s}}+\frac{\chi_{K}(p) \boldsymbol{\alpha}([p])}{p^{2 s}}\right)^{-1} .
$$

Возвращаясь к прообразу отображения $\boldsymbol{\alpha}$, мы получаем разложение

$$
\sum_{\mu=1}^{\infty} \frac{\boldsymbol{\tau}(\mu)}{\mu^{s}}=\prod_{p \text { простые }}\left([1]-\frac{\boldsymbol{\tau}(p)}{p^{s}}+\frac{\chi_{K}(p)[p]}{p^{2 s}}\right)^{-1}
$$

уточняющее разложение (2.38).

Рассмотрим теперь линейное расширение представления (3.15) до линейного представления кольца $\widetilde{\mathcal{H}}$ на инвариантном подпространстве

$$
\mathcal{I}_{k}=\mathcal{I}_{k}^{1}\left(Q_{1}, \ldots, Q_{h}\right)=\left\{\left(P_{1}, \ldots, P_{h}\right) \in \mathcal{P}_{k} \mid P_{i} \in \mathcal{I}_{k}\left(Q_{i}\right)\right\}
$$

пространства $\mathcal{P}_{k}=\mathcal{P}_{k}^{1}\left(Q_{1}, \ldots, Q_{h}\right)$ гармонических векторов рода 1 и веса $k$ (равного степени) относительно системы $Q_{1}, \ldots, Q_{h}$, где $\mathcal{I}_{k}\left(Q_{i}\right)=\mathcal{I}_{k}^{1}\left(Q_{i}\right)$ обозначает пространство вида (3.13) всех $\mathbf{E}_{i}$-инвариантных гармонических форм. Из предложения 3.1 легко следует, что пространство $\mathcal{P}_{k}^{1}\left(Q_{i}\right)$ всех гармонических форм рода 1 и степени $k$ относительно матрицы $Q_{i}$ состоит из линейных комбинаций с коэффициентами из $\mathbb{C}$ многочленов

$$
P_{i}^{k}(X)=\left(x_{1}-\gamma_{i} x_{2}\right)^{k} \quad \text { и } \quad P_{-i}^{k}(X)=\left(x_{1}-\bar{\gamma}_{i} x_{2}\right)^{k} \quad\left(X=\left(\begin{array}{l}
x_{1} \\
x_{2}
\end{array}\right)\right)
$$

где $\gamma_{i}$ определено в (5.2). Используя равенства (5.6), легко проверить, что эти многочлены являются $\mathbf{E}_{i}$-инвариантными в том и только том случае, если степень $k$ делится на порядок $e=e(\mathcal{O})=\#(E(\mathcal{O}))$ группы единиц кольца $\mathcal{O}$, что мы и будем предполагать в дальнейшем. В этом случае пространство $\boldsymbol{I}_{k}$ совпадает с пространством $\mathcal{P}_{k}$ всех гармонических векторов и $2 h$ векторов

$$
\mathbf{P}_{ \pm i}=\mathbf{P}_{ \pm i}^{(k)}=\left(0, \ldots, 0, P_{ \pm i}^{k}, 0, \ldots, 0\right) \quad \mathrm{c} \quad \pm i= \pm 1, \ldots, \pm h
$$

образуют базис этого пространства.

Образ формы $P \in \mathcal{I}_{k}\left(Q_{i}\right)$ под действием (3.16) оператора Гекке $\mid \tau_{i j}(D)$ с $D \in$ $A_{i j}(\mu)$ можно записать в виде

$$
P \mid \tau_{i j}(D)=\sum_{D^{\prime} \in \mathbf{E}_{i} \backslash \mathbf{E}_{i} D \mathbf{E}_{j}} P\left(D^{\prime} X\right)=P(D X),
$$


поскольку $\mathbf{E}_{i} D \mathbf{E}_{j}=\mathbf{E}_{i} D$. Если $P=P_{i}^{k}$ и $D=\left(\begin{array}{ll}a & b \\ c & d\end{array}\right)$, то, согласно и (5.5), мы получаем

$$
\begin{aligned}
P_{i}^{k} \mid \tau_{i j}(D) & =P_{i}^{k}(D X)=\left(a x_{1}+b x_{2}-\gamma_{i}\left(c x_{1}+b x_{2}\right)\right)^{k} \\
& =\left(a-\gamma_{i} c\right)^{k}\left(x_{1}-\frac{\gamma_{i} d-b}{a-\gamma_{i} c} x_{2}\right)^{k} \\
& =\left(a-\gamma_{i} c\right)^{k}\left(x_{1}-\gamma_{j} x_{2}\right)^{k}=\alpha_{i j}(D)^{k} P_{j}^{k} .
\end{aligned}
$$

Отсюда следует, что подпространство $\mathcal{P}_{k}^{+}=\mathcal{I}_{k}^{+}$пространства $\mathcal{P}_{k}$, натянутое на векторы $\mathbf{P}_{1}, \ldots, \mathbf{P}_{h}$, инвариантно относительно всех операторов Гекке из кольца $\widetilde{\mathcal{H}}$. В частности, используя (2.29), аналогично формуле (5.8) мы получаем для $i=1, \ldots, h$ и $\mu=1,2, \ldots$ формулу

$$
\mathbf{P}_{i} \mid \boldsymbol{\tau}(\mu)=\sum_{j=1}^{h} \boldsymbol{\alpha}_{i j}^{(k)}(\mu) \mathbf{P}_{j}, \quad \text { где } \quad \boldsymbol{\alpha}_{i j}^{(k)}(\mu)=\sum_{\substack{(\alpha) \subset \mathcal{M}_{i} \mathcal{M}_{j}^{-1}, N(\alpha)=\mu N\left(\mathcal{M}_{i} \mathcal{M}_{j}^{-1}\right)}} \alpha^{k} .
$$

Кроме того, по формуле (3.4) для операторов Гекке, отвечающих элементам (2.30), получаем соотношение

$$
\mathbf{P}_{i} \mid[d]=d^{k} \mathbf{P}_{i} \quad(i=1, \ldots, h) .
$$

Аналогично получаем формулы

$$
P_{-i}^{k} \mid \tau(D)=P_{-i}^{k}(D X)=\left(a-\bar{\gamma}_{i} c\right)^{k}\left(x_{1}-\bar{\gamma}_{j} x_{1}\right)^{k}=\bar{\alpha}_{i j}^{k}(D) \bar{P}_{-j}^{k}
$$

откуда следует, что подпространство $\mathcal{P}_{k}^{-}=\mathcal{I}_{k}^{-}$пространства $\mathcal{P}_{k}$, натянутое на векторы $\mathbf{P}_{-1}, \ldots, \mathbf{P}_{-h}$, инвариантно относительно всех операторов Гекке из кольца $\widetilde{\mathcal{H}}$. В частности, для $i=1, \ldots, h$ и $\mu=1,2, \ldots$ мы получаем формулы

$$
\mathbf{P}_{-i} \mid \boldsymbol{\tau}(\mu)=\sum_{j=1}^{h} \overline{\boldsymbol{\alpha}}_{i j}^{(k)} \mathbf{P}_{-j}
$$

где

$$
\overline{\boldsymbol{\alpha}}_{i j}^{(k)}(\mu)=\sum_{\substack{(\alpha) \subset \overline{\mathcal{M}}_{i}\left(\overline{\mathcal{M}}_{j}\right)^{-1}, N(\alpha)=\mu N\left(\overline{\mathcal{M}}_{i}\left(\overline{\mathcal{M}}_{j}\right)^{-1}\right)}} \alpha^{k}=\sum_{\substack{(\alpha) \subset \overline{\mathcal{M}}_{i} \overline{\mathcal{M}}_{j}^{-1}, N(\alpha)=\mu N\left(\overline{\mathcal{M}}_{i} \overline{\mathcal{M}}_{j}^{-1}\right)}} \bar{\alpha}^{k} .
$$

Таким образом, можно ограничиться рассмотрением операторов Гекке на пространстве $\mathcal{P}_{k}^{+}$. С этой целью мы введем линейное представление о полугруппы всех ненулевых идеалов кольца $\mathcal{O}$ на пространстве $\mathcal{P}_{k}^{+}$, полагая

$$
\mathbf{P}_{i} \circ \mathfrak{A}=\alpha_{i j}(\mathfrak{A})^{k} \mathbf{P}_{j}, \quad \text { если } \quad \mathfrak{A} \mathcal{M}_{i} \sim \mathcal{M}_{j} \Leftrightarrow \mathfrak{A} \mathcal{M}_{i}=\alpha_{i j}(\mathfrak{A}) \mathcal{M}_{j} \text { с } \alpha_{i j}(\mathfrak{A}) \in K .
$$

Обозначим через $M(\mathfrak{A})=\left(\alpha_{i j}(\mathfrak{A})^{k}\right)$ матрицу оператора о $\mathfrak{A}$, записанную в базисе $\mathbf{P}_{1}, \ldots, \mathbf{P}_{h}$. Тогда, очевидно, имеют место соотношения

$$
M(\mathfrak{A}) M(\mathfrak{B})=M(\mathfrak{A} \mathfrak{B}) \quad \text { и } \quad M((\alpha))=\alpha^{k} \cdot 1_{h}, \quad \text { если } \quad \alpha \in \mathcal{O},
$$


и матрица (5.21) оператора Гекке $\mid \boldsymbol{\tau}(\mu)$ может быть записана в том же базисе в виде

$$
\left(\boldsymbol{\alpha}_{i j}^{(k)}(\mu)\right)=\sum_{\mathfrak{A} \subset \mathcal{O}, N(\mathfrak{A})=\mu} M(\mathfrak{A}) .
$$

Если $\mathfrak{A} \mathcal{M}_{i}=\alpha_{i j}(\mathfrak{A}) \mathcal{M}_{j}$, то, переходя к сопряженным модулям, мы получаем соотношение $\overline{\mathfrak{A M}}_{i}=\bar{\alpha}_{i j}(\mathfrak{A}) \mathcal{M}_{j}$. Так как для каждого полного модуля $\mathcal{M}$ с кольцом множителей $\mathcal{O}$ имеет место соотношение $\mathcal{M} \overline{\mathcal{M}}=N(\mathcal{M}) \mathcal{O}$, где $N(\mathcal{M})$ -

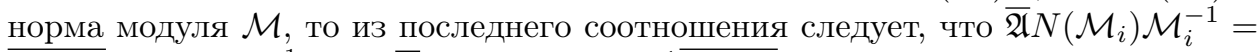
$\overline{\alpha_{i j}(\mathfrak{A})} N\left(\mathcal{M}_{j}\right) \mathcal{M}_{j}^{-1}$, или $\overline{\mathfrak{A}} \mathcal{M}_{j}=N\left(\mathcal{M}_{i}\right)^{-1} \overline{\alpha_{i j}(\mathfrak{A})} N\left(\mathcal{M}_{j}\right) \mathcal{M}_{i}$, откуда по определению матриц $M$ имеет место соотношение

$$
M(\overline{\mathfrak{A}})=N^{-2 t} \bar{M}(\mathfrak{A}) N^{2}, \quad \text { где } \quad N=\operatorname{diag}\left(\sqrt{N\left(\mathcal{M}_{1}\right)}, \ldots, \sqrt{N\left(\mathcal{M}_{h}\right)}\right) .
$$

Это соотношение можно переписать в виде

$$
M^{\prime}(\overline{\mathfrak{A}})=N M(\overline{\mathfrak{A}}) N^{-1}=N^{-1}{ }^{t} \bar{M}(\mathfrak{A}) N={ }^{t}\left(N \bar{M}(\mathfrak{A}) N^{-1}\right)={ }^{t} \bar{M}^{\prime}(\mathfrak{A}) .
$$

С другой стороны, на основании (5.23), матрицы $M^{\prime}(\mathfrak{A})$ вместе с матрицами $M(\mathfrak{A})$ коммутируют друг с другом и, в частности, коммутируют с матрицей $M^{\prime}(\overline{\mathfrak{A}})$. Таким образом, каждая из матриц $M^{\prime}(\mathfrak{A})=N M(\mathfrak{A}) N^{-1}$ является нормальной и по хорошо известной теореме линейной алгебры все матрицы $M^{\prime}(\mathfrak{A})$ могут быть одновременно диагонализированы. Отсюда следует, что существует базис пространства $\mathcal{P}_{k}^{+}$, состоящий из общих собственных векторов всех операторов о․ Пусть $\mathbf{F}=a_{h} \mathbf{P}_{1}+\cdots+a_{h} \mathbf{P}_{h}-$ один из таких собственных векторов, так что

$$
\mathbf{F} \circ \mathfrak{A}=\rho(\mathfrak{A}) \mathbf{F} \quad \text { для всех идеалов } \mathfrak{A} \subset \mathcal{O} .
$$

Так как любой идеал $\mathfrak{A} \subset \mathcal{O}$ в степени $h$ является главным идеалом, $\mathfrak{A}^{h}=\alpha \mathcal{O}$ с $\alpha \in \mathcal{O}$, то по (5.23) мы заключаем, что

$$
\rho(\mathfrak{A})^{h}=\rho\left(\mathfrak{A}^{h}\right)=\rho(\alpha \mathcal{O})=\alpha^{k}=\left(\frac{\alpha}{|\alpha|}\right)^{k}|\alpha|^{k}=\left(\frac{\alpha}{|\alpha|}\right)^{k} N(\mathfrak{A})^{k h / 2} .
$$

Отсюда следует, что

$$
\rho(\mathfrak{A})=\Psi_{k}(\mathfrak{A}) N(\mathfrak{A})^{k / 2} \quad \text { с характером Гекке } \Psi_{k}(\mathfrak{A})=\left(\sqrt[h]{\frac{\alpha}{|\alpha|}}\right)^{k} .
$$

Согласно формулам (5.21) и (5.24), собственные числа операторов Гекке $\mid \boldsymbol{\tau}(\mu)$ на пространстве $\mathcal{P}_{k}^{+}$, отвечающие собственному вектору $\mathbf{F}$, можно записать в виде

$$
\rho(\boldsymbol{\tau}(\mu))=\sum_{\mathfrak{A} \subset \mathcal{O}, N(\mathfrak{A})=\mu} \rho(\mathfrak{A}),
$$

и соответствующая дзета-функция равна

$$
\begin{aligned}
Z(s, \mathbf{F}) & =\sum_{\mu=1}^{\infty} \frac{\rho(\boldsymbol{\tau}(\mu))}{\mu^{s}}=\sum_{\mathfrak{A} \subset \mathcal{O}} \frac{\rho(\mathfrak{A})}{N(\mathfrak{A})^{s}}=\sum_{\mathfrak{A} \subset \mathcal{O}} \frac{\Psi_{k}(\mathfrak{A})}{N(\mathfrak{A})^{s-k / 2}} \\
& =\prod_{\text {простые идеалы } \mathfrak{P} \subset \mathcal{O}}\left([1]-\frac{\Psi_{k}(\mathfrak{P})}{N(\mathfrak{P})^{s-k / 2}}\right)^{-1}=\zeta_{\mathcal{O}}\left(s-k / 2, \Psi_{k}\right),
\end{aligned}
$$


где

$$
\zeta_{\mathcal{O}}\left(s, \Psi_{k}\right)=\sum_{\mathfrak{A}} \frac{\Psi_{k}(\mathfrak{A})}{N(\mathfrak{A})^{s}}=\prod_{\mathfrak{P}}\left(1-\frac{\Psi_{k}(\mathfrak{P})}{N(\mathfrak{P})^{s}}\right)^{-1}
$$

с $\mathfrak{A}$ и $\mathfrak{P}$, пробегающими все ненулевые идеалы и все простые идеалы кольца $\mathcal{O}$ соответственно, является дзета-функцией Гекке кольца $\mathcal{O}$ с характером $\Psi_{k}$. Отсюда следует, что ряд Дирихле $Z(s, \mathbf{F})$ сходится абсолютно и равномерно в каждой правой полуплоскости $\operatorname{Re} s \geqslant 1+k / 2+\varepsilon$ с положительным $\varepsilon$. Функция $Z(s, \mathbf{F})$ снова может быть представлена в каждой области абсолютной сходимости посредством интеграла Меллина от тета-суммы вида $\Theta(z ; \mathbf{F}, Q)=$ $a_{1} \theta\left(z ; P_{1}, Q_{1}\right)+\cdots+a_{h} \theta\left(z ; P_{h}, Q_{h}\right)$ для некоторой матрицы $Q$, эквивалентной одной из матриц $Q_{i}$ :

$$
r(2 ; \mathbf{F}) Z(s, \mathbf{F})=\frac{(2 \pi)^{s}}{\Gamma(s)} \int_{0}^{\infty}(\Theta(\sqrt{-1} t ; \mathbf{F}, Q)-\mathbf{F}(0)) t^{s-1} d t,
$$

где $r(2 ; \mathbf{F})$ и $\mathbf{F}(0)$ обозначают коэффициент при $e^{2 \pi \sqrt{-1} z}$ и постоянный член разложения Фурье тета-суммы соответственно и где $\Gamma(s)$ - гамма-функция. Это представление позволяет доказать, что дзета-функция имеет мероморфное аналитическое продолжение на всю $s$-плоскость и удовлетворяет функциональному уравнению. Альтернативно, аналитические свойства дзета-функции $\zeta_{\mathcal{O}}\left(s, \Psi_{k}\right)$ можно исследовать, используя анализ Фурье на пространстве аделей поля $\mathbb{Q}(\sqrt{-d})$, как это было сделано в диссертации Тэйта [22].

\section{Список литературы}

[1] А.Н. Андрианов, "О дзета-функциях ортогональных групп одноклассных положительно-определенных квадратичных форм”, Алгебра и анализ, 17:4 (2005), $3-41$.

[2] S. Rallis, "Langlands' functoriality and Weil representation", Amer. J. Math., 104:3 (1982), 469-515.

[3] А.Н. Андрианов, "Квадратичные сравнения и рациональность локальных дзетарядов тернарных и кватернарных квадратичных форм", Алгебра и анализ, 6:2 (1994), 3-52.

[4] А.Н. Андрианов, "Композиция решений квадратичных диофантовых уравнений", УМН, 46:2 (1991), 3-40.

[5] A. N. Andrianov, Queen's lectures on arithmetical composition of quadratic forms, Queen's Papers in Pure and Appl. Math., 92, Queen's Univ., Kingston, ON, 1992.

[6] А.Н. Андрианов, "Гармонические тета-функции и операторы Гекке", Алгебра и анализ, 8:5 (1996), 1-31.

[7] А. Н. Андрианов, "Мультипликативные свойства решений квадратичных диофантовых задач", Алгебра и анализ, 2:1 (1990), 3-46.

[8] J. W. S. Cassels, Rational quadratic forms, London Math. Soc. Monogr., 13, Academic Press, London, 1978.

[9] A. N. Andrianov, Quadratic forms and Hecke operators, Grundlehren Math. Wiss., 286, Berlin, Springer-Verlag, 1987.

[10] А.Н. Андрианов, "Мультипликативные разложения целочисленных представлений бинарных квадратичных форм", Алгебра и анализ, 5:1 (1993), 81-108.

[11] M. Kashiwara, M. Vergne, "On the Segal-Shale-Weil representations and harmonic polynomials", Invent. Math., 44:1 (1978), 1-47. 
[12] E. Freitag, Singular modular forms and theta relations, Lecture Notes in Math., 1487, Springer-Verlag, Berlin, 1991.

[13] A. Ogg, Modular forms and Dirichlet series, W. A. Benjamin, New York, 1969.

[14] А.Н. Андрианов, Г. Н. Малолеткин, "Поведение тета-рядов рода $n$ при модулярных подстановках", Изв. АН СССР. Сер. матем., 39:2 (1975), 243-258.

[15] А.Н. Андрианов, "Симметрии гармонических тета-функций целочисленных квадратичных форм", УМН, 50:4 (1995), 3-44.

[16] А.Н. Андрианов, "Сингулярные кольца Гекке-Шимуры и операторы Гекке на зигелевых модулярных формах", Алгебра и анализ, 11:6 (1999), 1-68.

[17] А. Н. Андрианов, А. А. Панчишкин, "Сингулярные операторы Фробениуса на зигелевых модулярных формах с характерами и дзета-функции”, Алгебра и анализ, 12:2 (2000), 64-99.

[18] A. N. Andrianov, "On diagonalisation of singular Frobenius operators on Siegel modular forms", Amer. J. Math., 125:1 (2003), 139-165.

[19] A. O. L. Atkin, J. Lehner, "Hecke operators om $\Gamma_{0}(m)$ ", Math. Ann., 185:2 (1970), 134-160.

[20] W.-C. W. Li, "Newforms and functional equations", Math. Ann., 212:4 (1975), 285315.

[21] З. И. Боревич, И. Р. Шафаревич, Теория чисел, Наука, М., 1964.

[22] J. Tate, Fourier analysis in number fields and Hecke's zeta functions, Ph.D. Thesis, Princeton Univ., Princeton, NJ, 1950.

А. Н. Андрианов (А. N. Andrianov)

Санкт-Петербургское отделение

Математического института им. В. А. Стеклова РАН

E-mail: anandr@pdmi.ras.ru
Поступила в редакцию 06.05.2006 